\title{
Collider phenomenology of a unified leptoquark model
}

\author{
T. Faber, ${ }^{*}$ Y. Liu, ${ }^{\dagger}$ and W. Porod ${ }^{*}$ \\ Institut für Theoretische Physik und Astrophysik, Universität Würzburg, D-97074 Würzburg, Germany \\ M. Hudec $\odot^{\S}$ and M. Malinskýø\|l \\ Institute for Particle and Nuclear Physics, Charles University, 18000 Praha 8, Czech Republic \\ F. Staub \\ Institute for Nuclear Physics, Karlsruhe Institute of Technology, D-76131 Karlsruhe, Germany \\ H. Kolešová $\odot^{* *}$ \\ Department of Mathematics and Physics, University of Stavanger, 4021 Stavanger, Norway
}

(Received 9 May 2019; revised manuscript received 19 November 2019; accepted 6 April 2020; published 19 May 2020)

\begin{abstract}
We demonstrate that in a recently proposed unified leptoquark model based on the gauge group $S U(4)_{C} \times S U(2)_{L} \times U(1)_{R}$ significant deviations from the Standard Model values of $R_{K}$ and $R_{K^{*}}$ can be accommodated without any need of extra heavy fermions. Low energy data, in particular lepton-flavorviolating $\mu$ decays and $K_{L} \rightarrow e \mu$, severely constrain the available parameter space. We show that in the allowed part of the parameter space (i) some of the lepton-flavor-violating tau decay branching ratios are predicted to be close to their current experimental limits. (ii) The underlying scalar leptoquarks can be probed at the LHC via their dominant decay modes into tau leptons and electrons and the third generation quarks. (iii) The constraints from meson oscillations imply that the masses of scalar gluons, another pair of colored multiplets around, have to be bigger than around $15 \mathrm{TeV}$, and thus, they can be probed only at a future $100 \mathrm{TeV}$ collider. In both neutral and charged variants, these scalars decay predominantly into third generation quarks, with up to $O(10 \%)$ branching ratios into family-mixed final states. Besides that, we comment on the phenomenology of the scalar gluons in the current scenarios in the case that the $B$-decay anomalies eventually disappear.
\end{abstract}

DOI: 10.1103/PhysRevD.101.095024

\section{INTRODUCTION}

The latest results of the LHC clearly show that the Standard Model (SM) continues to be a remarkably successful description of nature. So far, only a handful of experimental observations show deviations from its predictions. At the moment, exciting direct hints of physics beyond the SM are the recently observed anomalies in $B$-meson decays [1-6], which suggest lepton flavor

\footnotetext{
thomas.faber@physik.uni-wuerzburg.de yang.liu@uni-wuerzburg.de *porod@physik.uni-wuerzburg.de

\$hudec@ipnp.troja.mff.cuni.cz

"malinsky@ipnp.troja.mff.cuni.cz

ๆflorian.staub@kit.edu

***helena.kolesova@uis.no
}

Published by the American Physical Society under the terms of the Creative Commons Attribution 4.0 International license. Further distribution of this work must maintain attribution to the author(s) and the published article's title, journal citation, and DOI. Funded by SCOAP ${ }^{3}$. universality violation (LFUV) in the ratios $R_{K^{(*)}}=\Gamma(\bar{B} \rightarrow$ $\left.\bar{K}^{(*)} \mu^{+} \mu^{-}\right) / \Gamma\left(\bar{B} \rightarrow \bar{K}^{(*)} e^{+} e^{-}\right)$and $R_{D^{(*)}}=\Gamma\left(\bar{B} \rightarrow D^{(*)} \tau \bar{\nu}\right) /$ $\Gamma\left(\bar{B} \rightarrow D^{(*)} l \bar{\nu}\right),(l=e, \mu)$, with, e.g., [7]

$$
R_{K}=0.846_{-0.054}^{+0.060}(\text { stat })_{-0.014}^{+0.016}(\text { syst })
$$

Assuming that these anomalies are not a result of experimental systematics, they can be accounted for by leptoquarks (LQs) of various kinds [8-22]. However, building models in which these fields emerge from the extended gauge symmetries is generally rather challenging, especially in the light of very stringent constraints on lepton flavor violation (LFV) from various experimental searches; see, e.g., $[23,24]$.

Several attempts to build UV complete SM extensions of this kind already exist in the literature [18,25-36]. Most of them aim at getting the vector leptoquark $U_{1}$ (cf. [37]), transforming as $(3,1,+2 / 3)$ under the SM gauge group $G_{\mathrm{SM}}=S U(3)_{c} \times S U(2)_{L} \times U(1)_{Y}$, sufficiently light as it is an excellent candidate to explain the anomalies. It emerges naturally from the breaking of 
TABLE I. Fermion and scalar content of the model at the $G=S U(4)_{C} \otimes S U(2)_{L} \otimes U(1)_{R}$ and $G_{\mathrm{SM}}$ levels, respectively.

\begin{tabular}{ll}
\hline \hline Fermions & Scalars \\
\hline$F_{L(4,2,0)}=\left(\begin{array}{c}Q \\
L\end{array}\right)$ & $\chi_{(4,1,+1 / 2)}=\left(\begin{array}{c}\bar{S}_{1(3,1,+2 / 3)}^{\dagger} \\
\chi_{(1,1,0)}^{0}\end{array}\right)$ \\
$f_{R(4,1,+1 / 2)}^{u}=\left(\begin{array}{c}u_{R} \\
\nu_{R}\end{array}\right)$ & $H_{(1,2,+1 / 2)}$ \\
$f_{R(4,1,-1 / 2)}^{d}=\left(\begin{array}{c}d_{R} \\
e_{R}\end{array}\right)$ & $\Phi_{(15,2,+1 / 2)}=\left(\begin{array}{cc}G_{(8,2,+1 / 2)}+\frac{1}{\sqrt{12}} H_{2} & R_{2(3,2,+7 / 6)} \\
\tilde{R}_{2(\overline{3}, 2,-1 / 6)}^{\dagger} & \frac{-3}{\sqrt{12}} H_{2(1,2,+1 / 2)}\end{array}\right)$ \\
$N_{L(1,1,0)}$ & \\
\hline \hline
\end{tabular}

$S U(4)_{C}$ to $S U(3)_{c}$ which fixes the properties of $U_{1}$ up to effects from generation mixing of the fermions to which it couples. However, in most of these works the details of the scalar sector, e.g., the masses and couplings of the scalars, have been ignored.

To this end, we have recently [38] presented a detailed analysis of a model $[39,40]$ featuring an $S U(4)_{C} \times$ $S U(2)_{L} \times U(1)_{R}$ gauge symmetry and a minimal fermionic content in which the freedom in the scalar sector in principle allows for an explanation of $R_{K^{(*)}}$ even with the $U_{1}$ mass above $1000 \mathrm{TeV}$ (as required by the stringent $K_{L}$ decay constraints). In doing so, we have used the $S O(10)$-inspired simplifying assumption that all Yukawa couplings are symmetric in the flavor indices (in the defining basis). This hypothesis, however, turned out to be too restrictive as it does not resolve the tension between $R_{K}$ and the bounds on $K_{L} \rightarrow e \mu$.

In this paper we show that when releasing the symmetry conditions on the Yukawa matrices significant deviations from the SM values of $R_{K^{(*)}}$ in the direction indicated by the experiment can be accommodated without violating any other experimental bound. In the scheme under consideration, the scalar leptoquark $R_{2}$ couples dominantly to the electrons; needless to say, in such a case one cannot address the discrepancies observed in the angular distributions of the decay $B \rightarrow K^{*} \mu^{+} \mu^{-}$[41]. On the other hand, the allowed parameter space is quite restricted which implies that the properties of the additional scalars are fixed to a high degree. Consequently, this leads to rather specific predictions for LHC searches.

The paper is organized as follows: in Sec. II we summarize the main features of the model with a particular focus on its aspects relevant for the $B$-physics anomalies. In Sec. III we discuss various constraints stemming from the low energy data and their consequences for the properties of the new scalars. This is followed by a discussion of the resulting collider phenomenology in Sec. IV. A brief summary is given in Sec. V.

For our investigation we used the SARAH package [42-46] which needed to be extended considerably. We present this extension in Appendix C. For the numerical calculations we used the generated model files to produce a spectrum generator based on SPheno $[47,48]$. For the calculation of cross sections at hadron colliders we have used the SARAH-generated interface to MadGraph_aMC@NLO [49,50].

\section{MODEL ASPECTS}

We briefly summarize here the main features of the model that are important for the subsequent discussion. For further details we refer to Refs. [38-40]. The model is based on the gauge group $G=S U(4)_{C} \otimes S U(2)_{L} \otimes U(1)_{R}$ where the $\mathrm{SM} S U(3)_{c}$ emerges as part of the $S U(4)_{C}$ factor. In this class of models, leptons (including the right-handed neutrinos) are unified with quarks in representations of $G$ as summarized in Table I. The sub-eV neutrino masses and the observed leptonic mixing pattern are accommodated via an inverse seesaw mechanism [51] by adding three extra generations of a gauge-singlet fermion $N_{L}$ to the original model of Ref. [39] as proposed in [40]. The inverse seesaw is the only source of the lepton number violation while the baryon number remains a good symmetry to all orders in perturbation theory [38].

\section{A. Symmetry breaking and scalar sector}

The scalar sector consists of three irreducible representations of $G$ as given in Table I. At the level of $G_{\mathrm{SM}}$, the colorless part includes a complex singlet $\chi^{0}$ and two Higgs doublets $\mathrm{H}$ and $\mathrm{H}_{2}$. The gauge symmetry is broken by their vacuum expectation values (VEVs) in two steps

$$
G \stackrel{\left\langle\chi^{0}\right\rangle,\left\langle H_{2}\right\rangle}{\longrightarrow} G_{\mathrm{SM}} \stackrel{\langle H\rangle,\left\langle H_{2}\right\rangle}{\longrightarrow} S U(3)_{c} \otimes U(1)_{Q} .
$$

We parametrize the VEVs as

$$
\left\langle\chi^{0}\right\rangle=\frac{v_{\chi}}{\sqrt{2}}, \quad\langle H\rangle=\frac{\sin \beta}{\sqrt{2}}\left[\begin{array}{c}
0 \\
v_{\mathrm{ew}}
\end{array}\right], \quad\left\langle H_{2}\right\rangle=\frac{\cos \beta}{\sqrt{2}}\left[\begin{array}{c}
0 \\
v_{\mathrm{ew}}
\end{array}\right],
$$

where the square brackets denote the $S U(2)_{L}$ doublet structure, $v_{\text {ew }}=246 \mathrm{GeV}$ and $v_{\chi} \approx 1000 \mathrm{TeV}$. The latter is chosen such that the vector leptoquark mass is consistent 
with the stringent bound ${ }^{1}$ set by the nonobservation of $K_{L} \rightarrow e \mu$.

As usual in the two-Higgs-doublet models (2HDM), it is convenient to rotate the $S U(2)$ doublets via

$$
\left(\begin{array}{c}
\hat{H} \\
h
\end{array}\right)=\left(\begin{array}{cc}
\cos \beta & -\sin \beta \\
\sin \beta & \cos \beta
\end{array}\right)\left(\begin{array}{c}
H \\
H_{2}
\end{array}\right),
$$

where $h$ accommodates the entire electroweak VEV and contains also the would-be Nambu-Goldstone bosons to be eaten by $W^{ \pm}$and $Z$, whereas $\hat{H}$ is a second Higgs doublet which does not participate at the electroweak symmetry breaking. One can follow the analogy with the 2HDMs one step further. In particular, the physical component of the $h$ field defined by transformation (4) corresponds almost exactly to the SM Higgs because the current setting may be viewed as the $2 \mathrm{HDM}$ in the decoupling regime as the $\hat{H}$ mass is expected to be pushed up to the $S U(4)_{C}$ breaking scale $v_{\chi}$. Furthermore, the admixture of $\chi^{0}$ in the physical Higgs is also suppressed by $v_{\text {ew }} / v_{\chi}$. All this can be readily verified by the analysis of the most general renormalizable scalar potential ${ }^{2}$

$$
\begin{aligned}
V= & \mu_{H}^{2}|H|^{2}+\mu_{\chi}^{2}|\chi|^{2}+\mu_{\Phi}^{2} \operatorname{Tr}\left(|\Phi|^{2}\right)+\lambda_{1}|H|^{2}|\chi|^{2}+\lambda_{2}|H|^{2} \operatorname{Tr}\left(|\Phi|^{2}\right)+\lambda_{3}|\chi|^{2} \operatorname{Tr}\left(|\Phi|^{2}\right) \\
& +\left(\lambda_{4} H_{i}^{\dagger} \chi^{\dagger} \Phi^{i} \chi+\text { H.c. }\right)+\lambda_{5} H_{i}^{\dagger} \operatorname{Tr}\left(\Phi_{j}^{\dagger} \Phi^{i}\right) H^{j}+\lambda_{6} \chi^{\dagger} \Phi^{i} \Phi_{i}^{\dagger} \chi+\lambda_{7}|H|^{4}+\lambda_{8}|\chi|^{4}+\lambda_{9} \operatorname{Tr}\left(|\Phi|^{4}\right) \\
& +\lambda_{10}\left(\operatorname{Tr}|\Phi|^{2}\right)^{2}+\left(\lambda_{11} H_{i}^{\dagger} \operatorname{Tr}\left(\Phi^{i} \Phi^{j}\right) H_{j}^{\dagger}+\lambda_{12} H_{i}^{\dagger} \operatorname{Tr}\left(\Phi^{i} \Phi^{j} \Phi_{j}^{\dagger}\right)+\lambda_{13} H_{i}^{\dagger} \operatorname{Tr}\left(\Phi^{i} \Phi_{j}^{\dagger} \Phi^{j}\right)+\text { H.c. }\right) \\
& +\lambda_{14} \chi^{\dagger}|\Phi|^{2} \chi+\lambda_{15} \operatorname{Tr}\left(\Phi_{i}^{\dagger} \Phi^{j} \Phi_{j}^{\dagger} \Phi^{i}\right)+\lambda_{16} \operatorname{Tr}\left(\Phi_{i}^{\dagger} \Phi^{j}\right) \operatorname{Tr}\left(\Phi_{j}^{\dagger} \Phi^{i}\right)+\lambda_{17} \operatorname{Tr}\left(\Phi_{i}^{\dagger} \Phi_{j}^{\dagger}\right) \operatorname{Tr}\left(\Phi^{i} \Phi^{j}\right) \\
& +\lambda_{18} \operatorname{Tr}\left(\Phi_{i}^{\dagger} \Phi_{j}^{\dagger} \Phi^{i} \Phi^{j}\right)+\lambda_{19} \operatorname{Tr}\left(\Phi_{i}^{\dagger} \Phi_{j}^{\dagger} \Phi^{j} \Phi^{i}\right),
\end{aligned}
$$

where $|H|^{2}=H_{i}^{\dagger} H^{i},|\chi|^{2}=\chi^{\dagger} \chi,|\Phi|^{2}=\Phi_{i}^{\dagger} \Phi^{i}$ with $i$ and $j$ denoting the $S U(2)_{L}$ indices; matrix notation has been used to capture the $S U(4)_{C}$ structure and the traces run only over the $S U(4)_{C}$ indices.

The colored scalar degrees of freedom are the $\bar{S}_{1}$ field originating from $\chi$ which dominates the Goldstone mode associated with the vector leptoquark, an $S U(2)_{L}$ doublet $G$ of charged and neutral scalar gluons and two other leptoquark doublets $R_{2}$ and $\tilde{R}_{2}$, all of them stemming from $\Phi$.

Although we have chosen $v_{\chi}$ so large that the effects of the extra vector bosons (the $Z^{\prime}$ and the vector leptoquark $U_{1}$ ) are completely negligible, the model allows for a certain part of the scalar spectrum being much lighter. This can easily be seen by neglecting for the moment the effects of the $S U(2)_{L}$ breaking VEVs in the masses of the different components of the $\Phi$-field, ${ }^{3}$

$$
\begin{gathered}
m_{G}^{2}=\left(\frac{\sqrt{3} \lambda_{4}}{4} \tan \beta-\frac{3}{8}\left(\lambda_{6}+\lambda_{14}\right)\right) v_{\chi}^{2}, \\
m_{R_{2}}^{2}=\left(\frac{\sqrt{3} \lambda_{4}}{4} \tan \beta+\frac{\lambda_{14}-3 \lambda_{6}}{8}\right) v_{\chi}^{2},
\end{gathered}
$$

\footnotetext{
${ }^{1}$ This bound can actually be lowered by more than an order of magnitude if one maximally exploits the freedom in the associated unitary charged-current interaction matrix [52]; however, in the current study we need to save this freedom for configuring the scalar leptoquark interactions.

${ }^{2}$ The completeness of formula (5) can readily be verified by computer codes such as sym2int [53].

${ }^{3}$ Needless to say, the weak isospin mass splitting for a heavy doublet $X$ is only of the order $\delta m_{X}=O\left(v_{\mathrm{ew}}^{2} / m_{X}\right) \lesssim 10 \mathrm{GeV}$.
}

$$
\begin{gathered}
m_{\tilde{R}_{2}}^{2}=\left(\frac{\sqrt{3} \lambda_{4}}{4} \tan \beta+\frac{\lambda_{6}-3 \lambda_{14}}{8}\right) v_{\chi}^{2}, \\
m_{\hat{H}}^{2}=\frac{\sqrt{3} \lambda_{4}}{2 \sin (2 \beta)} v_{\chi}^{2}
\end{gathered}
$$

where $\mu_{\Phi}^{2}$ has been eliminated using the minimization conditions for the potential. This yields an approximate tree-level sum rule [38]

$$
m_{G}^{2}+2 m_{\hat{H}}^{2} \sin ^{2} \beta=\frac{3}{2}\left(m_{R_{2}}^{2}+m_{\tilde{R}_{2}}^{2}\right) .
$$

It is well known that, unlike $\tilde{R}_{2}$, the $R_{2}$ leptoquark has the potential to simultaneously accommodate $R_{K}<1$ and $R_{K^{*}}<1$ [54]. From Eq. (10) one can see that $R_{2}$ can be in the $\mathrm{TeV}$ range even in the case of a rather large $v_{\chi}$ if there is an appropriate fine-tuning between $\lambda_{4} \tan \beta, \lambda_{6}$, and $\lambda_{14}$ such that the entire bracket in Eq. (7) is suppressed to the $\mathcal{O}\left(10^{-6}\right)$ level.

Assuming for the moment that $\lambda_{4}$ is at least of the order of $10^{-2}$, one can see from Eq. (10) that relatively light scalar gluons are possible in scenarios where $R_{2}$ is light and $\tilde{R}_{2}$ heavy. We will thus also investigate such scenarios. In principle $\lambda_{4}$ could also be smaller yielding somewhat lighter $\hat{H}$ and $\tilde{R}_{2}$ states. However, the contribution of $\tilde{R}_{2}$ to lepton-flavor-violating observables implies that the masses should be in the multi-TeV range. For completeness, we note that the large number of parameters allows one to obtain easily a SM-like Higgs boson with $m_{h^{0}}=125 \mathrm{GeV}$. Since the purely scalar interaction vertices play a negligible 
TABLE II. Summary of the sample input values used in the numerical analysis of Secs. III and IV (unless stated otherwise). Note also that all other BSM scalars have masses of the order $\mathcal{O}\left(m_{A}\right)$.

\begin{tabular}{lc}
\hline \hline \multicolumn{2}{c}{ Numerical input values } \\
\hline$Y_{2}$ & $\operatorname{diag}\left(10^{-8}, 10^{-7}, 10^{-5}\right)$ \\
$Y_{5}$ & $\operatorname{diag}\left(10^{-2}, 5 \times 10^{-2}, 10^{-1}\right)$ \\
$v_{\chi}$ & $4 \times 10^{6} \mathrm{GeV}$ \\
$m_{A}, m_{R_{2}}$ & $2 \times 10^{5} \mathrm{GeV}, 1500 \mathrm{GeV}$ \\
$\cos \beta$ & 0.02 \\
\hline \hline
\end{tabular}

role in the phenomenology under consideration, we shall not specify the particular choices of $\lambda$ 's there.

\section{B. Fermionic sector}

The fermion masses are generated by the following Lagrangian:

$$
\begin{aligned}
-\mathcal{L}_{\mathcal{Y}}= & \overline{f_{R}^{u}} Y_{1} H F_{L}+\overline{f_{R}^{u}} Y_{2} \Phi F_{L}+\overline{f_{R}^{d}} Y_{3} H^{\dagger} F_{L} \\
& +\overline{f_{R}^{d}} Y_{4} \Phi^{\dagger} F_{L}+\overline{f_{R}^{u}} Y_{5} \chi N_{L}+\frac{1}{2} N_{L}^{T} \mathcal{C}_{\mu} N_{L}+\text { H.c. }
\end{aligned}
$$

where $Y_{i}$ are matrices of Yukawa couplings and $\mu$ is a Majorana mass matrix. Without loss of generality, we work in a basis where the charged-lepton mass matrix is flavor diagonal. The up- and down-type quarks in the mass basis are given by $\hat{q}_{L}=V_{q} q_{L}$ and $\hat{q}_{R}=U_{q} q_{R}$ for $q=u$, $d$, with the four arbitrary unitary matrices in the flavor space being constrained by $V_{\mathrm{CKM}}=V_{u} V_{d}^{\dagger}$.

Two of the Yukawa matrices above are strongly related to the masses of down-type quarks and charged leptons, namely

$$
\begin{gathered}
U_{d}^{\dagger} \hat{M}_{d} V_{d}=\left(\frac{\sin \beta}{\sqrt{2}} Y_{3}+\frac{\cos \beta}{2 \sqrt{6}} Y_{4}\right) v_{\mathrm{ew}}, \\
\hat{M}_{e}=\left(\frac{\sin \beta}{\sqrt{2}} Y_{3}-\frac{3 \cos \beta}{2 \sqrt{6}} Y_{4}\right) v_{\mathrm{ew}},
\end{gathered}
$$

where $\hat{M}_{u, d, e}$ are diagonal matrices of the corresponding fermion masses.

The Yukawa interactions of the LQs and scalar gluons are encoded solely in $Y_{2}$ and $Y_{4}$. Equations (12) and (13) determine $Y_{4}$ up to the two rotation matrices. On the other hand, due to the extended neutrino sector, the other important matrix $Y_{2}$, as well as $Y_{5}$, can be chosen essentially arbitrarily. Indeed, the measured up-type quark masses satisfying

$$
U_{u}^{\dagger} \hat{M}_{u} V_{\mathrm{CKM}} V_{d}=\left(\frac{\sin \beta}{\sqrt{2}} Y_{1}+\frac{\cos \beta}{2 \sqrt{6}} Y_{2}\right) v_{\mathrm{ew}}
$$

can always be attained by a suitable choice of $Y_{1}$. The light Majorana neutrino mass matrix, from which the neutrino masses and mixing matrix (PMNS) follow, can then be obtained via a proper choice of the Majorana mass matrix $\mu$.

While both $Y_{2}$ and $Y_{4}$ do in general contribute to various lepton-flavor-violating processes, only $Y_{4}$ is relevant for a tree-level explanation of the $R_{K^{(*)}}$ anomalies. For simplicity, we will assume that all elements of $Y_{2}$ are negligibly small (see Table II for our particular choice of the SPheno input). As will be clarified in Sec. III, the main reason for this is the need to satisfy the very stringent limits on the LFV muon decays ( $\mu \rightarrow e \gamma$ and $\mu \rightarrow 3 e$; see [38]) as well as other constraints such as those coming from $\tau \rightarrow e \pi^{0}$; in this respect, the situation with $Y_{4}$ alone is much "safer" than that of any significant interplay among the two. It is also worth noting that in this case the specific form of $U_{u}$ is not important as vertices where $Y_{4}$ appears do not contain righthanded up quarks. The only other sector it affects is neutrinos; there it enters together with $Y_{5}$ which, however, entertains a lot of freedom anyway. For definiteness, in what follows we set $U_{u}=U_{d} V_{\mathrm{CKM}}^{\dagger}$. Note also that, unless specified otherwise, the results below (especially those of Sec. IVA) are quite robust with respect to invoking small but nonzero $Y_{2}$.

\section{RARE LEPTON AND MESON DECAYS}

As explained in Sec. II, we assume that the only relatively light beyond-Standard-Model (BSM) field around is the leptoquark doublet $R_{2}$ and all other heavy fields are effectively decoupled. Cases with other relatively light extra multiplets are discussed at the end of this section. As outlined in the previous section we assume that $Y_{2}$ is small and, thus, the only relevant BSM matter interactions are those following from the term proportional to $Y_{4}$. For the $R_{2}$ leptoquark, these read

$\mathcal{L}_{R_{2}}=\overline{\hat{d}}_{L} \hat{Y}_{4}^{d e} \hat{e}_{R} R_{2}^{+2 / 3}+\overline{\hat{u}}_{L} V_{\mathrm{CKM}} \hat{Y}_{4}^{d e} \hat{e}_{R} R_{2}^{+5 / 3}+$ H.c.,

with the relevant Yukawa matrices parametrized as

$$
\begin{aligned}
\hat{Y}_{4}^{d e} & =\left(\begin{array}{lll}
y_{d e} & y_{d \mu} & y_{d \tau} \\
y_{s e} & y_{s \mu} & y_{s \tau} \\
y_{b e} & y_{b \mu} & y_{b \tau}
\end{array}\right), \\
\hat{V}_{\mathrm{CKM}} \hat{Y}_{4}^{d e} & =\left(\begin{array}{lll}
y_{u e} & y_{u \mu} & y_{u \tau} \\
y_{c e} & y_{c \mu} & y_{c \tau} \\
y_{t e} & y_{t \mu} & y_{t \tau}
\end{array}\right) .
\end{aligned}
$$

\section{A. Constraints on the $\hat{\boldsymbol{Y}}_{4}$ structure}

Without referring to the specific pattern of the matrix above imposed by the extended symmetry of the model 
[cf. Eqs. (12) and (13)] several simple but important observations can be made.

First, the interactions in Eq. (15) involve the righthanded leptons. In view of $R_{K}$, this implies that the corresponding tree-level contributions to $C_{9}$ and $C_{10}$ (entering at the scale where the leptoquarks are integrated out) have not only the same magnitude but also the same sign. Since the $\mathrm{SM}$ contribution yields $C_{9}^{\mathrm{SM}} \approx-C_{10}^{\mathrm{SM}}$ there is only a very small interference between the NP and the SM contributions in the $b \rightarrow s l^{+} l^{-}$amplitudes. Notice that there are ways to circumvent this feature by making the loop contributions dominant (see $[55,56]$ ); this, however, is not applicable in the current scenario.

Second, interactions in Eq. (15) generally induce new sources of LFUV whenever two columns of $\hat{Y}_{4}^{d e}$ differ. In this respect, $R_{K}<1$ can be achieved if and only if the LQs couple more to the electrons than to the muons [54], i.e., when $\left|y_{s e} y_{b e}\right|>\left|y_{s \mu} y_{b \mu}\right|$.

The third point is that the interactions in Eq. (15) mediate LFV processes whenever there are nonzero entries of $\hat{Y}_{4}^{\text {de }}$ in two different columns. For example, very stringent constraints arise from the experimental limits on $\operatorname{BR}\left(K_{L}^{0} \rightarrow\right.$ $\left.e^{ \pm} \mu^{\mp}\right) \propto\left|y_{s e} y_{d \mu}^{*}+y_{d e} y_{s \mu}^{*}\right|^{2}$ or from $\mu \rightarrow e \gamma$ whose amplitudes are given by linear combinations of $y_{q e} y_{q^{\prime} \mu}^{*}$. To this end, it is clear that all the muon number violating processes mediated by $R_{2}$ will be suppressed if

$$
y_{d \mu}=y_{s \mu}=y_{b \mu}=0
$$

approximately holds.

As indicated earlier, $\hat{Y}_{4}^{d e}$ cannot be chosen arbitrarily in our model as it is a subject of the extended symmetry constraints. In particular, applying the flavor rotations defined below Eq. (11) and using relations (12) and (13) one obtains the following pattern [57]:

$$
\hat{Y}_{4}^{d e}=\frac{\sqrt{3 / 2}}{v_{\mathrm{ew}} \cos \beta}\left(\hat{M}_{d} U_{d}-V_{d} \hat{M}_{e}\right)
$$

with $U_{d}$ and $V_{d}$ being arbitrary unitary matrices. The question now is whether this structure is compatible with $R_{K}$ significantly smaller than 1 and a suppressed LFV pattern.

In Ref. [38], this model was studied under an extra $S O(10)$ inspired assumption $V_{d}=U_{d}^{*}$ and with all possible phases neglected in a second step. In such a case, the interaction matrix in Eq. (18) simplifies to

$$
\hat{Y}_{4}^{d e}=\sqrt{\frac{3}{2}} \frac{\sqrt{1+\tan ^{2} \beta}}{v_{\text {ew }}}\left(\begin{array}{ccc}
V_{11}\left(m_{\mathrm{d}}-m_{\mathrm{e}}\right) & V_{12}\left(m_{\mathrm{d}}-m_{\mu}\right) & V_{13}\left(m_{\mathrm{d}}-m_{\tau}\right) \\
V_{21}\left(m_{\mathrm{s}}-m_{\mathrm{e}}\right) & V_{22}\left(m_{\mathrm{s}}-m_{\mu}\right) & V_{23}\left(m_{\mathrm{s}}-m_{\tau}\right) \\
V_{31}\left(m_{\mathrm{b}}-m_{\mathrm{e}}\right) & V_{32}\left(m_{\mathrm{b}}-m_{\mu}\right) & V_{33}\left(m_{\mathrm{b}}-m_{\tau}\right)
\end{array}\right),
$$

where $V_{i j}$ denotes the elements of the $V_{d}$ mixing matrix. Clearly, the requirements such as Eq. (17) are in contradiction with the unitarity of $V_{d}$, and thus LFV is principally unavoidable. In [38] it was found (by scanning over the considered parameter space) that the experimental bound $\operatorname{BR}\left(K_{L} \rightarrow \mu e\right)<4.7 \times 10^{-12}$ [58] inevitably leads to $R_{K} \geq 1$, at odds with measurements.

Consequently, this implies that the assumption $V_{d}=U_{d}^{*}$ is inconsistent with the requirement of simultaneously explaining $R_{K}$ and respecting the bound from the $K_{L} \rightarrow$ $\mu e$ decay. However, such a model assumption is only fully justified at the scale where one still has the left-right symmetry which, however, is broken well above $v_{\chi}$ (see, e.g., [59] and references therein for explicit constructions), and renormalization group effects will lead to a breaking of $V_{d}=U_{d}^{*}$ anyway. We also note that the current model might not emerge from $S O(10)$ but from another framework.

In the general case of $V_{d} \neq U_{d}^{*}$ we have the freedom to choose 6 angles and 12 phases. In order to suppress the muon number violating processes we require the conditions (17) to be satisfied to a high precision (at least to the order of $m_{e} / v_{\text {ew }} \cos \beta$ ), especially due to the very stringent limits on $\mu \rightarrow e \gamma, \mu \rightarrow e e e$, and $K_{L} \rightarrow \mu e$. The general form of $U_{d}$ and $V_{d}$ conforming this requirement can be found in Appendix A.

Two of the remaining three angles therein can be subsequently constrained by invoking the stringent upper limits on the lepton-flavor-violating $\tau$ decays, along with the desire to maintain non-negligible $\Delta R_{K^{(*)}}$. In the part of the parameter space with the best potential to fulfill these requirements the Yukawa matrix of Eq. (18) takes the form

$\hat{Y}_{4}^{d e} \simeq \frac{\sqrt{3 / 2}}{v_{\mathrm{ew}} \cos \beta}\left(\begin{array}{ccc}0 & 0 & m_{\tau} e^{i \delta_{4}} \sin \phi \\ m_{\mathrm{s}} e^{i \delta_{1}} / \sqrt{2} & 0 & m_{\tau} e^{i \delta_{5}} \cos \phi \\ m_{b} e^{i \delta_{2}} / \sqrt{2} & 0 & -m_{b} e^{i \delta_{3}} / \sqrt{2}\end{array}\right)$

as detailed in Appendix A. It is parametrized by a single angle $\phi \in\langle 0, \pi\rangle$ and five phases $\delta_{i} \in\langle 0,2 \pi\rangle$ which, in turn, define what we call the "sweet spot" region.

In this part of the parameter space the experimentally preferred values of $R_{K}$ and $R_{K^{*}}$ call for

$$
m_{R_{2}} \cos \beta \simeq 20 \mathrm{GeV} \text {, }
$$




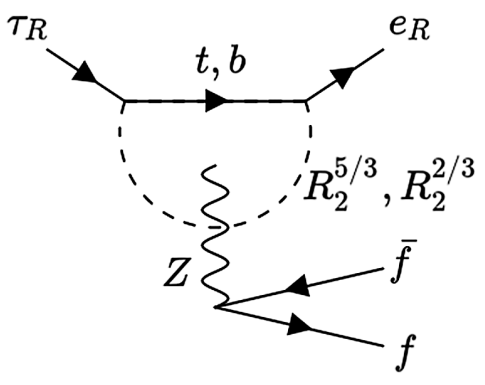

FIG. 1. The dominant interactions mediating the lepton-flavorviolating $\tau$ decays.

regardless of the choice of $\phi$ and $\delta_{i}$ 's. Hence, one needs $\cos \beta \ll 1$ in order to obey the bounds from direct leptoquark searches. Since this requirement, together with Eq. (20), selects a rather special part of the parameter space, the question arises in which other channels such a setting can be tested. There are essentially two broad classes of these, namely, the low energy observables and the LHC signals. We will focus here on the low energy part first and discuss the collider aspects in the next section.

\section{B. Predictions and smoking gun signals}

From the construction it is clear that there will be no additional constraints from any muon number violating decays such as $\mu \rightarrow e \gamma$. In fact, we can achieve any value of $\operatorname{BR}(\mu \rightarrow e \gamma)$ between zero and the experimental bound by arranging small deviations from the extreme scenario Eq. (17) with essentially no impact on the findings below. In contrast, at the same time we cannot avoid sizable effects in the $\tau$ sector.

\section{Tau decays}

The leptoquarks contribute to the $\tau \rightarrow e \gamma$ and $\tau \rightarrow e e e$ decays at the loop level, whereas to the final states involving mesons already at the tree level. Nevertheless, we find that, due to the differences in the magnitudes of relevant Yukawas, the $Z$ penguins (see Fig. 1) induced by the third generation quarks dominate over the tree-level contributions also for the $e \pi^{+} \pi^{-}$and $e K^{+} K^{-}$final states.

A somewhat less important contribution arises from the photon penguin, where in Fig. 1 the $Z$ gets replaced by $\gamma$. We have collected the relevant formulas for the $\gamma$ and $Z$ contributions in Appendix B. We find that the photon contribution comes with an overall factor of $1 / m_{R_{2}}^{2}$ and that of the $Z$ penguin with a factor $1 / m_{Z}^{2}$. We note for completeness that, nevertheless, the structure of the loop functions is such that all these vanish in the limit of $m_{R_{2}} \rightarrow \infty$. We have also found that the box contributions are subdominant.

For the evaluation of the predictions we have extended the Flavor-Kit package [60] to include decays of the $\tau$ lepton into an electron and two mesons. For the calculation of $\operatorname{BR}\left(\tau \rightarrow e \pi^{+} \pi^{-}\right)$we have adopted the formulas of Refs. $[61,62]$. In the case of the $e \pi^{\mp} K^{ \pm}$final states we

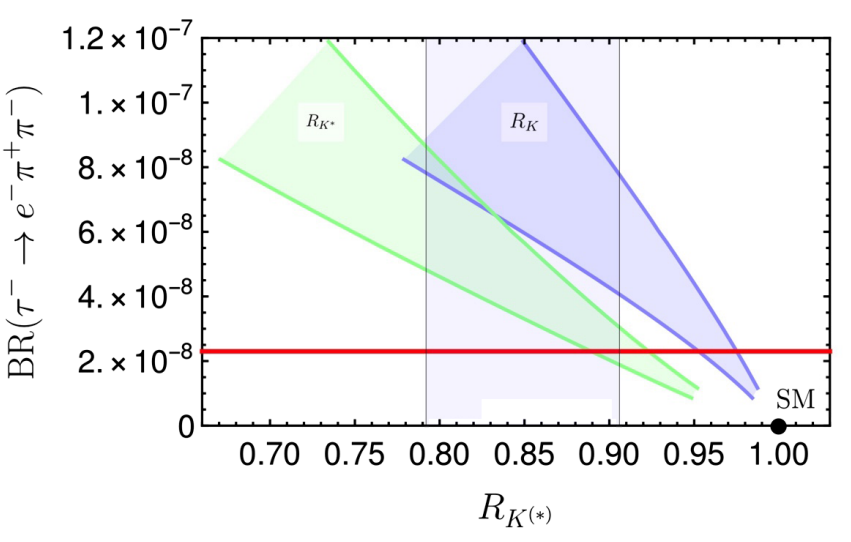

FIG. 2. Correlations between $\operatorname{BR}\left(\tau \rightarrow e \pi^{+} \pi^{-}\right)$and $R_{K}$ as well as $R_{K^{*}}$. The input parameters have been chosen as in Table II but $m_{R_{2}}$ has been varied between 0.9 and $2 \mathrm{TeV}$. Moreover, the quark mixing angles have been varied within the sweet spot region given by Eq. (20) yielding the bands shown. The red horizontal line gives the current bound $\operatorname{BR}\left(\tau \rightarrow e \pi^{+} \pi^{-}\right) \leq 2.3 \times 10^{-8}$ whereas the vertical band indicates the currently preferred range of Ref. [7]: $0.792 \leq R_{K} \leq 0.906$.

have taken the form factor given in [63] but used updated values for the corresponding meson masses. For the calculation of the $B$-meson observables we have employed FLAVIO [64] to which the data have been transferred via the WCXF interface [65].

The most stringent additional constraint stems from $\tau \rightarrow e \pi^{+} \pi^{-}$. In Fig. 2 we show $\operatorname{BR}\left(\tau \rightarrow e \pi^{+} \pi^{-}\right)$versus $R_{K}$ where we have taken the parameters as given in Table II except for $m_{R_{2}}$, which we have varied from $900 \mathrm{GeV}$ to $1.5 \mathrm{TeV}$, and scanned over all the sweet spot parameters of Eq. (20). Note that any digression from the sweet spot setting, i.e., diverting $\alpha$ and $\gamma$ of Appendix A from their optimal values (A6), generally pushes the resulting $\operatorname{BR}\left(\tau \rightarrow e \pi^{+} \pi^{-}\right)$up. One observes that there is a clear tension between the currently preferred value for $R_{K}$ [cf. Eq. (21)] and the bound on $\operatorname{BR}\left(\tau \rightarrow e \pi^{+} \pi^{-}\right)$which excludes scenarios with $m_{R_{2}} \cos \beta \lesssim 30 \mathrm{GeV}$. Hence, in what follows we adopt the limit case $m_{R_{2}} \cos \beta=30 \mathrm{GeV}$ (see Table II) and calculate predictions for the other relevant decay rates of $\tau$-lepton, as summarized in Table III.

The branching ratio for the final state containing $K^{+} K^{-}$ is smaller by roughly a factor of 2 , whereas those with $\pi^{ \pm} K^{\mp}$ are significantly more suppressed due to the flavorconserving quark current coupled to $Z$ in the relevant penguin. We also find that the other flavor violating $\tau$ decays are close to their experimental bound and within the sweet spot region vary only in a small range; see Table III. The dominance of the above-mentioned $Z$ penguin shows up also in the predictions for $\operatorname{BR}\left(\tau \rightarrow e \mu^{+} \mu^{-}\right)$and $\operatorname{BR}(Z \rightarrow$ $\left.e^{ \pm} \tau^{\mp}\right)$ which vary in the ranges $(1-1.3) \times 10^{-9}$ and $(3.4-4.4) \times 10^{-9}$, respectively. We note that the predicted range $\operatorname{BR}(\tau \rightarrow e \gamma)$ of a few times $10^{-9}$ provides a test of the current scenario at Belle II which aims to improve the limit 
TABLE III. Experimental bounds on various branching ratios $\operatorname{BR}(\tau \rightarrow X)$ [58] and corresponding ranges in the sweet spot region (main text) for the parameters given in Table II.

\begin{tabular}{lccccc}
\hline \hline$X$ & Bound & \multicolumn{1}{c}{ Range } & $X$ & Bound & Range \\
\hline$e \gamma$ & $3.3 \times 10^{-8}$ & $3.1 \times 10^{-9}-3.8 \times 10^{-9}$ & $e e^{+} e^{-}$ & $2.7 \times 10^{-8}$ & $1.2 \times 10^{-9}-1.6 \times 10^{-9}$ \\
$e \pi^{0}$ & $8 \times 10^{-8}$ & $1.4 \times 10^{-9}-4 \times 10^{-9}$ & $e \pi^{+} \pi^{-}$ & $2.3 \times 10^{-8}$ & $1.9 \times 10^{-8}-2.8 \times 10^{-8}$ \\
$e K_{S}$ & $2.6 \times 10^{-8}$ & $7.7 \times 10^{-11}-5.8 \times 10^{-11}$ & $e K^{+} K^{-}$ & $3.4 \times 10^{-8}$ & $5.9 \times 10^{-9}-8.5 \times 10^{-9}$ \\
$e \phi$ & $3.1 \times 10^{-8}$ & $1.2 \times 10^{-9}-1.9 \times 10^{-9}$ & $e \pi^{+} K^{-}$ & $3.7 \times 10^{-8}$ & $1.2 \times 10^{-20}-2.3 \times 10^{-11}$ \\
\hline \hline
\end{tabular}

on this channel to $3.3 \times 10^{-10}$ [66]. The model thus predicts that some lepton-flavor-violating $\tau$ decays should be discovered soon.

We note for completeness that in the allowed parameter space the flavor violating $\tau$ decays into muons are strongly suppressed, and thus, the observation of $\tau \rightarrow 3 \mu$ would rule out this scenario.

\section{Meson decays and oscillations}

We have also checked that the prediction for meson decays such as $b \rightarrow s \gamma, B \rightarrow K \tau e, B \rightarrow K \tau^{+} \tau^{-}$, or $B_{s} \rightarrow$ $\tau^{+} \tau^{-}$are fully consistent with the current experimental data. In the context of leptoquarks a potentially constraining observable is the ratio $\mathrm{BR}\left(K^{+} \rightarrow e^{+} \nu\right) /$ $\operatorname{BR}\left(K^{+} \rightarrow \mu^{+} \nu\right)$. However, due to the required smallness of $Y_{2}$, all leptoquark effects on observables with neutrinos in the final state are suppressed, and thus, this is also consistent with data.

Staying in this part of the parameter space we have also checked whether the low energy data can constrain the masses of the other components of $\Phi$. Our construction implies that the scalar gluons, both the charged and the neutral one, have flavor mixing couplings to quarks. This means in particular that the neutral one contributes at the tree level to $K^{0}-\bar{K}^{0}$ and $B_{q}-\bar{B}_{q}(q=d, s)$ mixing. We find that within the experimental and theoretical uncertainties $B_{s}-\bar{B}_{s}$ requires $m_{G^{0}} \gtrsim 10 \mathrm{TeV}$, whereas in the case of the $K^{0}-\bar{K}^{0}$ mixing the bound is $m_{G^{0}} \gtrsim 15 \mathrm{TeV}$. It might be surprising that the $K^{0}-\bar{K}^{0}$ mixing limit is only slightly more stringent than the $B$-meson one; this is a consequence of the specific shape of the parameter space considered here. We have also checked that loop-induced contributions to the $\Delta F=2$ transitions do not provide additional constraints on the allowed parameter space. We note, for completeness, that in other parts of the parameter space this bound increases up to $m_{G^{0}} \gtrsim 120 \mathrm{TeV}$.

\section{COLLIDER PHENOMENOLOGY}

\section{A. Collider phenomenology in the presence of flavor anomalies}

In the previous section we have found a restricted region of the parameter space where a significant effect in $R_{K^{(*)}}$ can be accommodated while staying consistent with the constraints from other flavor observables such as $\mu \rightarrow e \gamma$ and $K_{L} \rightarrow e \mu$. Here we shall discuss interesting collider signatures emerging in this part of the parameter space. Note that Eq. (10) allows for the situations where, apart from $R_{2}$, also the scalar gluons $G$, or even the whole scalar sector arising from $\Phi$, can be light enough to be tested either at the LHC or a prospective $100 \mathrm{TeV} p$ p-collider.

Remarkably enough, in the slice of the parameter space under consideration the leptoquarks have rather special properties. In particular, the pattern of their Yukawa couplings (20) is reflected in their decays. For the charge $2 / 3$ particle one finds, regardless of which point in the sweet spot region is chosen,

$$
\begin{aligned}
\mathrm{BR}\left(R_{2}^{+2 / 3} \rightarrow e^{+} b\right) \simeq & \mathrm{BR}\left(R_{2}^{+2 / 3} \rightarrow \tau^{+} b\right) \\
\simeq & \frac{m_{b}^{2}}{2 m_{\tau}^{2}}\left(\mathrm{BR}\left(R_{2}^{+2 / 3} \rightarrow \tau^{+} d\right)\right. \\
& \left.+\mathrm{BR}\left(R_{2}^{+2 / 3} \rightarrow \tau^{+} s\right)\right),
\end{aligned}
$$

where $\frac{m_{b}^{2}}{2 m_{\tau}^{2}} \simeq 1.17$ is calculated at the scale $m_{R_{2}}$. All other decay channels into charged leptons are negligible. Numerically, the BRs above amount to roughly $35 \%$ for the $e+j_{b}$ and $\tau+j_{b}$ final states and some $30 \%$ for the $\tau+j_{\text {light }}$ in the case of $Y_{2} \simeq 0$, and scale down appropriately if $R_{2}^{+2 / 3}$ might decay into other channels such as $R_{2}^{+2 / 3} \rightarrow \bar{\nu} t$ due to possible nonzero entries in $Y_{2}$.

Due to the hierarchical structure of the quark mixing matrix (CKM), a similar pattern appears for the charge $5 / 3$ particle where, in the case of $Y_{2} \simeq 0$, the non-negligible decay channels satisfy

$$
\begin{aligned}
\mathrm{BR}\left(R_{2}^{+5 / 3} \rightarrow t e^{+}\right) \simeq & \mathrm{BR}\left(R_{2}^{+5 / 3} \rightarrow t \tau^{+}\right) \\
\simeq & \frac{m_{b}^{2}}{2 m_{\tau}^{2}}\left(\mathrm{BR}\left(R_{2}^{+5 / 3} \rightarrow u \tau^{+}\right)\right. \\
& \left.+\mathrm{BR}\left(R_{2}^{+5 / 3} \rightarrow c \tau^{+}\right)\right) .
\end{aligned}
$$

These particles are searched for by the ATLAS [67] and CMS [68] experiments. Assuming branching ratios of $100 \%$ into a specific channel such as $\tau b$, bounds up to $1.1 \mathrm{TeV}$ have been set if the leptoquarks are pair produced. Since, however, various combinations of different decay channels involving different generations of fermions are allowed in the current scenario, the actual bounds are 
somewhat weaker. We have implemented both analyses in the CheckMate framework $[69,70]$ and found that collider searches constrain the $R_{2}$ mass only to about $890 \mathrm{GeV}$. This clearly shows that the setting discussed in Sec. III is fully consistent with bounds from direct searches. The decays of the $R_{2}^{+5 / 3}$ to $t$ quarks give rise to missing energy if the $W$ stemming from the $t$ decays leptonically. Therefore, we checked in addition whether any of the supersymmetry (SUSY) searches implemented in CheckMate can constrain our scenario. We find that although some of the analyses do indeed show some sensitivity to the corresponding final states, they do not exclude the current scenario. Potentially the high-luminosity phase of LHC may further constrain it but this requires a detailed study which is beyond the scope of this paper.

We now turn to the next component of $\Phi$ which can be potentially light, namely, the doublet of charged and neutral scalar gluons. In what follows we will neglect the splitting of $G^{0}$ into its scalar and pseudoscalar components since it is at most of $O(\mathrm{GeV})$. The scalar gluon interactions arising from $Y_{4}$ generally read

$$
\mathcal{L}_{G}=\left[G^{0} \hat{d}_{L}+G^{+} \overline{\hat{u}}_{L} V_{\mathrm{CKM}}\right] \hat{Y}_{4}^{d d} \hat{d}_{R}+\text { H.c. },
$$

where the relevant Yukawa matrix satisfies

$$
\hat{Y}_{4}^{d d}=\hat{Y}_{4}^{d e} U_{d}^{\dagger}=\frac{\sqrt{3 / 2}}{v_{\text {ew }} \cos \beta}\left(\hat{M}_{d}-V_{d} \hat{M}_{e} U_{d}^{\dagger}\right) .
$$

Note that the interactions of the scalar gluons with righthanded up-type quarks originate from $Y_{2}$ which, as mentioned earlier, is suppressed in our model. For this reason our findings differ significantly from the ones of Refs. [57,71,72]. Due to the $m_{b}$ enhancement in Eq. (25), the neutral scalar gluons are generally predicted to prefer decays to the $b$ quarks. In the sweet spot region discussed so far we obtain
$\hat{Y}_{4}^{d d} \simeq \frac{\sqrt{3 / 2}}{v_{\mathrm{ew}} \cos \beta}\left(\begin{array}{ccc}0 & m_{\tau} \sin \phi / \sqrt{2} & -m_{\tau} \sin \phi / \sqrt{2} \\ 0 & m_{\tau} \cos \phi / \sqrt{2} & -m_{\tau} \cos \phi / \sqrt{2} \\ -m_{\mu} & 0 & m_{b}\end{array}\right)$.

Here we have neglected all phases as their impact on the two-body decays is negligible. One finds the following ranges for the various branching ratios:

$$
\begin{aligned}
\operatorname{BR}\left(G^{0} \rightarrow b \bar{b}\right) & \simeq 0.7-0.75 \\
\operatorname{BR}\left(G^{0} \rightarrow b \bar{d}+d \bar{b}\right)+\operatorname{BR}\left(G^{0}\right. & \rightarrow b \bar{s}+s \bar{b}) \simeq 0.15, \\
\operatorname{BR}\left(G^{+} \rightarrow t \bar{b}\right) & \simeq 0.65-0.73
\end{aligned}
$$

The neutral states also have loop induced couplings to the gluons [73]. Denoting the scalar (pseudoscalar) component of $G^{0}$ by $\sigma^{0}\left(\phi^{0}\right)$, we find $\operatorname{BR}\left(\sigma^{0} \rightarrow g g\right) \simeq 0.05$ and $\operatorname{BR}\left(\phi^{0} \rightarrow g g\right) \simeq 0.01$. It has been noted already in Ref. [73] that the scalar contributions in the loop induced couplings are negligible even for $\lambda_{i}=1$, and thus, the parametric uncertainties due to the unknown $\lambda_{i}$ are tiny. The remaining decays are into two quarks of the first two generations. We found in the previous section that the mass of the scalar gluon should be above $\sim 15 \mathrm{TeV}$ due to the constraints on the $K^{0}-\bar{K}^{0}$ mixing. This is clearly too heavy for the LHC, and thus, one needs a $100 \mathrm{TeV} p p$-collider [74,75] to look for these states.

In Fig. 3 we present some of the dominant Feynman diagrams for the processes $p p \rightarrow G^{0} q \bar{q}(q=b, t)$ and $p p \rightarrow G^{+} b \bar{t}$, including also the contributions from the production of a scalar gluon pair with the subsequent decay of one of the scalar gluons into $q \bar{q}^{(\prime)}$. The corresponding cross sections for a $100 \mathrm{TeV}$ collider are shown in Fig. 4 where we have included all tree-level QCD contributions as well as all couplings of scalar gluons to quarks. The relevant Yukawa coupling $Y_{4}$ is chosen in the sweet spot region with $\phi=0$. For large scalar masses the

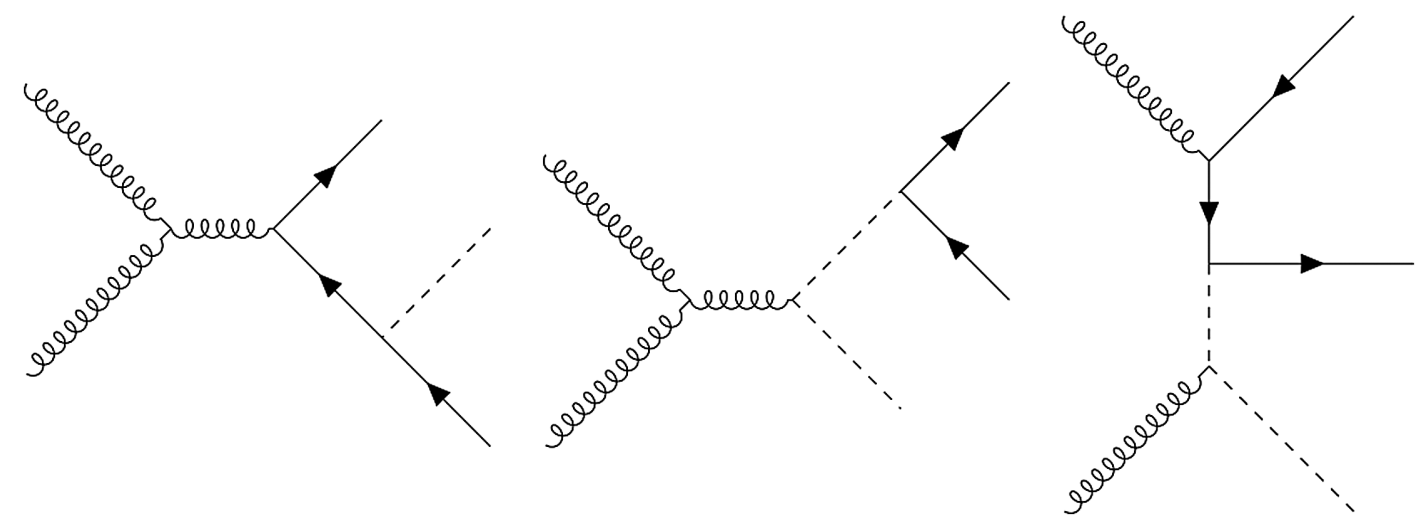

FIG. 3. Exemplary Feynman graphs for the dominant production cross sections $p p \rightarrow G^{0} q \bar{q}(q=b, t)$ and $p p \rightarrow G^{+} b \bar{t}$ at the hadron colliders. 


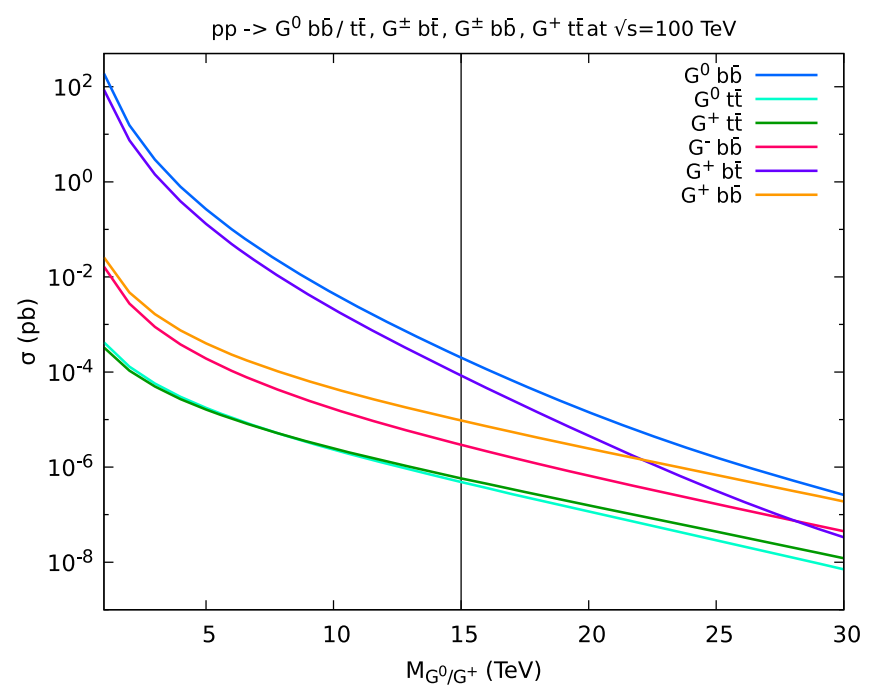

FIG. 4. Various production cross sections at a prospective $p p$ collider with $\sqrt{s}=100 \mathrm{TeV}$ as a function of the corresponding mass. In addition, the channel $G^{-} \bar{b} t$ also exists and neglecting the electroweak contributions one finds $\sigma\left(G^{-} \bar{b} t\right)=\sigma\left(G^{+} \bar{t} b\right)$. Here we have used the parameters given in Table II except for the masses of the scalar gluons. The vertical line indicates the bound on $m_{G^{0}} \simeq m_{G^{+}}$obtained from meson mixing.

production cross sections get a significant contribution also from the quark initial states or are even dominated by those. For instance, $\sigma\left(p p \rightarrow G^{+} b \bar{b}\right)$ varies by about $20 \%$ within the sweet spot region because of its dependence on $Y_{4}$. Note that the cross sections shown here are calculated at the tree level and we expect sizable QCD corrections. Combining the cross sections with the branching ratios above, we have found that the dominant signals will be in the $4 b$-jet and $2 t+2 b$-jets channels which are experimentally challenging.

\section{B. Scalar gluons at colliders without flavor anomalies}

Since the measurements of the $B$ anomalies still admit the case of being pure statistical fluctuations, in what follows we focus for completeness on the situation when both leptoquarks are too heavy to contribute significantly to the low energy observables and when the lightest BSM fields are the scalar gluons.

These particles are interesting on their own, and thus, we study here the limit case where all flavor violating couplings of the neutral scalar gluons are absent. This can be achieved, e.g., by setting $V_{d}$ and $U_{d}$ to the unit matrix which leads to

$$
\hat{Y}_{4}^{d d}=\sqrt{\frac{3}{2}} \frac{1}{v_{\mathrm{ew}} \cos \beta}\left(\hat{M}_{d}-\hat{M}_{e}\right) .
$$

Assuming that the elements of $Y_{2}$ are smaller at least by an order of magnitude compared to those in $Y_{4}$, the scalar gluons can have masses within the reach of the LHC. Consequently, Eq. (30) together with Eq. (24) imply that $\operatorname{BR}\left(G^{+} \rightarrow t \bar{b}\right)$ is close to one and that the neutral states decay dominantly into $b \bar{b}$.

The latter can also decay into two gluons. However, in this setting the neutral scalar gluons have suppressed couplings to the top-quark compared to the situations discussed for example in Refs. [39,73,76], which is due to the smallness of the $Y_{2}$ entries in the current scenario. Consequently, also the loop-induced $G^{0} g g$ coupling is
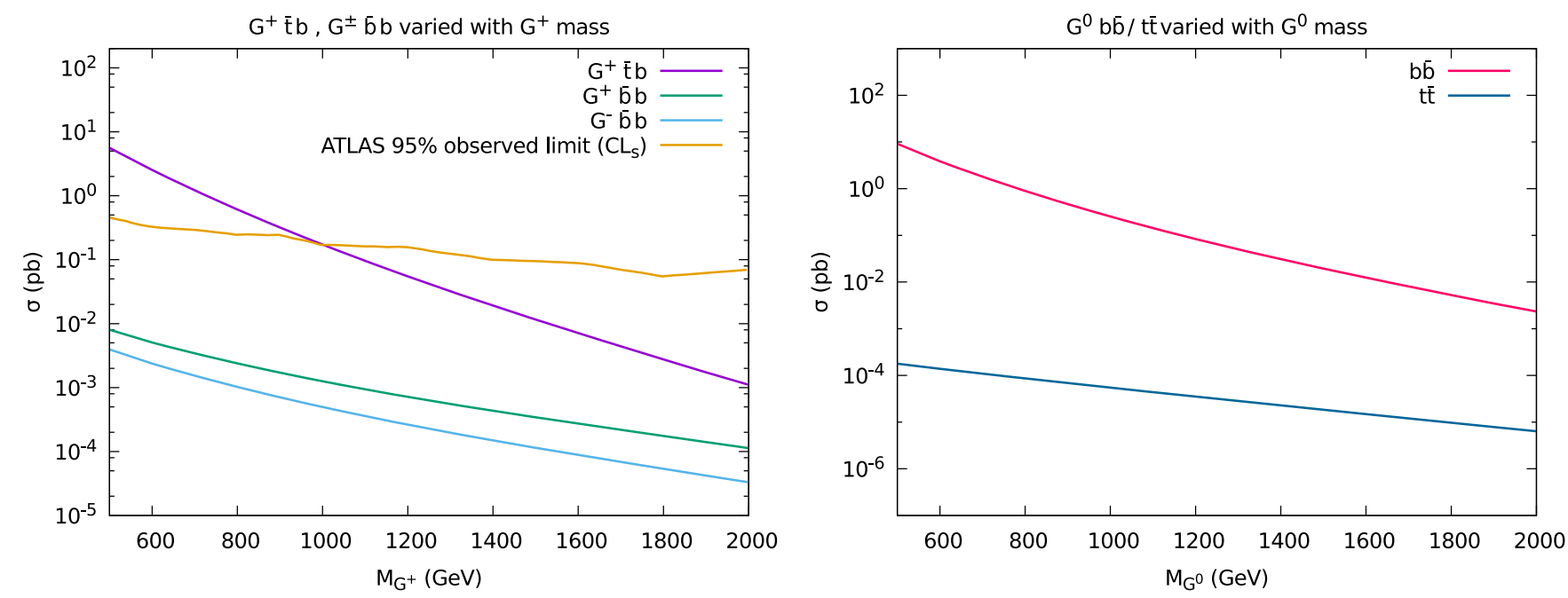

FIG. 5. Production cross sections at the LHC with $\sqrt{s}=13 \mathrm{TeV}$. On the left side the cross sections $\sigma\left(p p \rightarrow G^{+} \bar{t} b\right)$ (purple line), $\sigma\left(p p \rightarrow G^{+} \bar{b} b\right)$ (green line), and $\sigma\left(p p \rightarrow G^{-} \bar{b} b\right)$ (blue line) are shown as a function of $m_{G^{+}}$. The yellow line gives the current bound on the $G^{+} \bar{t} b$ final state obtained by the ATLAS experiment [80]. On the right side the cross sections $\sigma\left(p p \rightarrow G^{0} \bar{b} b\right)$ and $\sigma\left(p p \rightarrow G^{0} \bar{t} t\right)$ are shown as a function of $m_{G^{0}}$. 
significantly smaller compared to models where $Y_{2}$ induces large couplings to the $t$ quark. First, this implies that the decays into two gluons have a branching ratio of at most $5 \%$. Second, this also implies that the bounds from processes such as

$$
p p \rightarrow G^{0}+X \rightarrow 2 j+X
$$

(with $j$ being either a quark or a gluon jet) obtained by the CMS experiment $[77,78]$ do not constrain our model even when taking QCD corrections via a $K$ factor of 1.7 [79] into account. Instead, we have found that the strongest constraints come from the ATLAS search for the $H^{+} \bar{t} b$ production [80]. We can see from Fig. 5 that this excludes scenarios with $m_{G^{+}} \simeq 1 \mathrm{TeV}$. This is actually a conservative bound in the sense that we assume here $\operatorname{BR}\left(G^{+} \rightarrow\right.$ $t \bar{b})=1$ which maximizes the power of the experimental analysis. We want to stress that we have also included here the pair production $p p \rightarrow G^{+} G^{-}$combined with the subsequent decay $G^{-} \rightarrow \bar{t} b$. Due to the steep decrease of the cross sections with the mass this plot indicates that the reach of the LHC will not be above $1.5 \mathrm{TeV}$. We therefore show in Fig. 4 various cross sections at the $100 \mathrm{TeV}$ collider starting from masses in the $\mathrm{TeV}$ range which clearly shows that the cross sections in the low mass range is so large that these particles should be found within the first data sets.

\section{CONCLUSIONS}

In this paper we have studied a model based on the extended $S U(4)_{C} \times S U(2)_{L} \times U(1)_{R}$ gauge symmetry which is arguably the most minimal UV-complete gauge framework including vector and scalar leptoquark fields. It has been shown recently [38] that, among other features, this setup has the potential to accommodate significant effects in semileptonic $B$ decays. It is well known that, in this context, the strongest constraints stem from the nonobservation of $K_{L} \rightarrow e \mu$ and $\mu \rightarrow e \gamma$. In order for these to be satisfied along with $R_{K^{(*)}}$ well below 1 , a rather specific flavor pattern of the scalar leptoquark interactions with matter is required; for instance, all couplings of the supposedly light $R_{2}$ leptoquark to muons need to be strongly suppressed along with its couplings to the righthanded up-type quarks and left-handed charged leptons. We have shown that there exists a narrow region in the parameter space where a highly consistent picture can be achieved. This, in turn, leads to a very predictive scenario in which several other interesting phenomenological conclusions can be drawn.

First, there are sharp predictions for the branching ratios of $\tau \rightarrow e \pi^{+} \pi^{-}$and $\tau \rightarrow e K^{+} K^{-}$which are marginally compatible with the current experimental bounds; especially the first channel provides a strong constraint on the allowed parameter space. Moreover, also $\tau \rightarrow e \gamma$ and $\tau \rightarrow$ $3 e$ turn out to be close to their current experimental limits and, thus, their signals should be observable in the next round of experiments such as Belle II. Thus, if $R_{K}$ stays on its current value, nonobservation of the LFV $\tau$ decays will rule out the model as a whole.

Second, the charge- $2 / 3$ and $-5 / 3$ scalar leptoquarks, whose masses should not be much above $1 \mathrm{TeV}$ in order to address the $B$-anomalies, turn out to have rather specific decay properties which can be tested either at LHC or at a future $100 \mathrm{TeV} p p$ collider. In particular, we find that $\mathrm{BR}\left(R_{2}^{+2 / 3} \rightarrow e \bar{b}\right) \simeq \mathrm{BR}\left(R_{2}^{+2 / 3} \rightarrow \tau \bar{b}\right) \simeq \mathrm{BR}\left(R_{2}^{+2 / 3} \rightarrow \tau \bar{d}\right)+$ $\mathrm{BR}\left(R_{2}^{+2 / 3} \rightarrow \tau \bar{s}\right)$ and $\mathrm{BR}\left(R_{2}^{+5 / 3} \rightarrow t e^{+}\right) \simeq \mathrm{BR}\left(R_{2}^{+5 / 3} \rightarrow t \tau^{+}\right) \simeq$ $\mathrm{BR}\left(R_{2}^{+5 / 3} \rightarrow u \tau^{+}\right)+\mathrm{BR}\left(R_{2}^{+5 / 3} \rightarrow c \tau^{+}\right)$. As such, a clear indication should be expected in the high-luminosity LHC run if the mass of $R_{2}$ was in the indicated $1.5 \mathrm{TeV}$ ballpark.

Third, there is enough room in the allowed parameter space for relatively light scalar gluons (with electric charges 0 and 1) whose masses are constrained from meson mixing to be above some $15 \mathrm{TeV}$. Again, the branching ratios of their decays (including those into flavor violating channels) are fixed within narrow ranges which would facilitate their searches at future colliders.

Remarkably enough, the phenomenology of such relatively light scalar gluons in the model under consideration is interesting even if the $B$ anomalies eventually disappear. It turns out that in such a case the stringent limits from the meson mixing can be alleviated and the bounds on their masses can be lowered into the LHC domain. In this scenario the most stringent limit stems from the process $p p \rightarrow G^{+} \bar{t} b$ where we get a bound $m_{G} \simeq 1 \mathrm{TeV}$ recasting an ATLAS search for $H^{+}$. The usual bounds on $G^{0}$ do not apply in this model. In that situation the branching ratios into the third generation quarks, namely, $\mathrm{BR}\left(G^{+} \rightarrow t \bar{b}\right)$ and $\operatorname{BR}\left(G^{0} \rightarrow b \bar{b}\right)$, amount to almost $100 \%$.

\section{ACKNOWLEDGMENTS}

We thank Jorge Portoléz and Maria Jose Herrero Solans for useful discussion on $\tau$ decays into an $e^{-}$and two mesons and Vojtěch Pleskot for comments on the LHC leptoqurk production. F.S. is supported by the ERC Recognition Award ERC-RA-0008 of the Helmholtz Association. T. F., Y. L., and W. P. have been supported by the DFG, Project No. PO-1337/7-1. M. H. and M. M. acknowledge the support from the Grant agency of the Czech Republic, Project No. 20-17490S and from the Charles University Research Center UNCE/SCI/013. M. H. has also been supported by the Grant Agency of Charles University (GAUK) Project No. 1248119. H. K. has been supported by Grant No. PR-10614 within the ToppForsk-UiS program of the University of Stavanger and the University Fund. Finally, we acknowledge the comments of the referee which helped to discover an error in the original numerical code. 


\section{APPENDIX A: THE SWEET SPOT REGION}

We approximate Eq. (18) by neglecting $m_{e}$ and $m_{d}$, and further by neglecting the second generation masses when compared to the third ones. The resulting structure reads

$$
\hat{Y}_{4}^{d e}=\frac{\sqrt{3 / 2}}{v_{\mathrm{ew}} \cos \beta}\left(\begin{array}{ccc}
0 & -V_{12} m_{\mu} & -V_{13} m_{\tau} \\
U_{21} m_{s} & U_{22} m_{s}-V_{22} m_{\mu} & -V_{23} m_{\tau} \\
U_{31} m_{b} & V_{32} m_{b} & U_{33} m_{b}-V_{33} m_{\tau}
\end{array}\right) .
$$

As explained in the main text, we need to set the middle column, corresponding to the muon interactions, to zero. Within the current approximation scheme this is achieved if and only if the mixing matrices take the form

$$
\begin{gathered}
U_{d}=\left(\begin{array}{ccc}
e^{i \delta_{8}} \cos \gamma \sin \alpha & e^{i\left(\delta_{7}+\delta_{8}-\delta_{1}\right)} \cos \alpha & e^{i\left(\delta_{3}+\delta_{8}-\delta_{2}\right)} \sin \alpha \sin \gamma \\
-e^{i \delta_{1}} \cos \alpha \cos \gamma & e^{i \delta_{7}} \sin \alpha & -e^{i\left(\delta_{1}-\delta_{2}+\delta_{3}\right)} \cos \alpha \sin \gamma \\
-e^{i \delta_{2}} \sin \gamma & 0 & e^{i \delta_{3}} \cos \gamma
\end{array}\right), \\
V_{d}=\left(\begin{array}{ccc}
e^{i \delta_{9}} \cos \phi & 0 & -e^{i \delta_{4}} \sin \phi \\
-e^{i\left(-\delta_{4}+\delta_{5}+\delta_{9}\right)} \cos \alpha^{\prime} \sin \phi & e^{i \delta_{7} \sin \alpha^{\prime}} & -e^{i \delta_{5} \cos \phi \cos \alpha^{\prime}} \\
e^{i\left(-\delta_{4}+\delta_{6}+\delta_{9}\right)} \sin \phi \sin \alpha^{\prime} & e^{i\left(-\delta_{5}+\delta_{6}+\delta_{7}\right)} \cos \alpha^{\prime} & e^{i \delta_{6}} \cos \phi \sin \alpha^{\prime}
\end{array}\right),
\end{gathered}
$$

where $\alpha$ and $\alpha^{\prime}$ are related via $m_{s} \sin \alpha=m_{b} \sin \alpha^{\prime}$. This yields

$$
\hat{Y}_{4}^{d e}=\frac{\sqrt{3 / 2}}{v_{\mathrm{ew}} \cos \beta}\left(\begin{array}{ccc}
0 & 0 & e^{i \delta_{4}} m_{\tau} \sin \phi \\
-e^{i \delta_{1}} m_{s} \cos \alpha \cos \gamma & 0 & e^{i \delta_{5}} m_{\tau} \cos \phi \cos \alpha^{\prime} \\
-e^{i \delta_{2}} m_{b} \sin \gamma & 0 & e^{i \delta_{3}} m_{b} \cos \gamma-e^{i \delta_{6}} m_{\tau} \cos \phi \sin \alpha^{\prime}
\end{array}\right) .
$$

Note that only small $O\left(m_{\mu} / m_{b}\right)$ deviations from the displayed form of $U_{d}$ and $V_{d}$ are necessary in order to fulfill the condition (17) exactly. We always use those exact forms in the numerical calculations (SPheno) but, for the sake of clarity, stick within the approximation in (A2) and (A3) in all the equations in the text.

Another important restriction on the parameter space emerges from $\tau \rightarrow e \pi^{+} \pi^{-}$in which the tree-level $R_{2}^{+5 / 3}$ leptoquark contribution is suppressed with respect to the top- $R_{2}^{+5 / 3}$ induced $Z$ penguin. The latter is driven by the product of the 31 and 33 elements of $\left(V_{\mathrm{CKM}} \cdot \hat{Y}_{4}^{d e}\right)$ which, due to $V_{t b} \approx 1$, essentially coincide with the 31 and 33 elements of (A4). Hence, the ratio of the effective couplings governing $\Delta R_{K^{(*)}}$ and $\tau \rightarrow e \pi^{+} \pi^{-}$is roughly proportional to

$$
\frac{C_{\Delta R_{K^{(*)}}}}{C_{\tau \rightarrow e \pi^{+} \pi^{-}}} \propto \frac{y_{b e} y_{s e}}{y_{b e} y_{b \tau}}=\frac{-e^{i \delta_{1}} m_{\mathrm{s}} \cos \alpha \cos \gamma}{e^{i \delta_{3}} m_{\mathrm{b}} \cos \gamma-e^{i \delta_{6}} \frac{m_{s} m_{\tau}}{m_{b}} \cos \phi \sin \alpha} .
$$

As long as $\cos \gamma$ is non-negligible, the first term in the denominator of (A5) dominates and the right-hand side therein depends only on $\cos \alpha$ and an irrelevant overall phase. Hence, in order to maximize the effect in $R_{K^{(*)}}$ one should keep $\alpha \approx 0$. Note that the case of $\cos \gamma \sim 0$ is pathological as in this situation any sizable effect in $R_{K^{(*)}}$ relies on further enhancing $\left(m_{R} \cos \beta\right)^{-1}$ which either renders the 31 coupling of (A4) nonperturbative (for tiny $\cos \beta$ ) or requires very low $m_{R_{2}}$, at odds with direct searches.

To conclude, we shall fix

$$
\alpha=0, \quad \gamma=\pi / 4
$$

to maximize the effects in $R_{K^{(*)}}$. The remaining parameters are left free and span what we call the sweet spot region.

\section{APPENDIX B: DOMINANT CONTRIBUTIONS TO THE DECAY $\tau \rightarrow e \pi^{+} \pi^{-}$}

We collect here the formulas for the photon and the $Z$ penguins including leptoquarks for the decay $\tau \rightarrow e \pi^{+} \pi^{-}$. The corresponding matrix elements are given for the quark currents which then need to be hadronized according to the procedure presented in [61,63].

The matrix elements for the photon contribution reads

$$
\begin{aligned}
T_{\gamma, q}= & \bar{u}_{e}\left(p_{1}\right)\left[k^{2} \gamma_{\mu} A_{1}+i m_{\tau} \sigma_{\mu \nu} k^{\nu} A_{2}\right] P_{R} u_{\tau}(p) \\
& \times \frac{e^{2}}{k^{2}} e_{q} \bar{u}_{q}\left(p_{2}\right) \gamma^{\mu} v_{q}\left(p_{3}\right),
\end{aligned}
$$


with

$$
\begin{aligned}
& A_{1}=\frac{N_{c}}{576 \pi^{2}} \sum_{q} y_{q e}^{*} y_{q \tau} \frac{1}{m_{R_{2}}^{2}}\left(f\left(x_{q}\right)+e_{q} g\left(x_{q}\right)\right), \\
& A_{2}=\frac{N_{c}}{32 \pi^{2}} \sum_{q} y_{q e}^{*} y_{q \tau} \frac{1}{m_{R_{2}}^{2}}\left(\tilde{f}\left(x_{q}\right)+e_{q} \tilde{g}\left(x_{q}\right)\right),
\end{aligned}
$$

where $k$ is the photon 4-momentum, $e_{q}$ is the charge fraction of the corresponding quark, $N_{c}=3, y_{q l}$ 's are defined in Eq. (16), and we have neglected terms proportional to $m_{e} / m_{\tau}$. The sum runs over all quarks, with $x_{q}=\left(m_{q} / m_{R_{2}}\right)^{2}$. Note that in the case of $d$-type ( $u$-type) quarks, $m_{R_{2}}$ denotes the mass of the charge $2 / 3(5 / 3)$ leptoquark.

The loop functions above take the form

$$
\begin{gathered}
f(x)=\frac{2-9 x+18 x^{2}-11 x^{3}+6 x^{3} \log x}{(1-x)^{4}}, \\
g(x)=\frac{6(3-3 x+(2+x) \log x)}{(1-x)^{2}} \\
\tilde{f}(x)=\frac{1-6 x+3 x^{2}+2 x^{3}-6 x^{2} \log x}{6(1-x)^{4}} \\
\tilde{g}(x)=\frac{x^{2}-1-2 x \log x}{2(x-1)^{3}} .
\end{gathered}
$$

We have cross-checked that we can reproduce the formulas given in $[81,82]$.

The matrix elements for the $Z$-boson contribution read

$$
\begin{aligned}
T_{Z, q}= & \frac{1}{m_{Z}^{2}} \bar{u}_{e}\left(p_{1}\right) \gamma^{\mu} F_{R} P_{R} u_{\tau}(p) \bar{u}_{q}\left(p_{2}\right) \gamma_{\mu} \\
& \times\left(a_{L}^{q} P_{L}+a_{R}^{q} P_{R}\right) v_{q}\left(p_{3}\right),
\end{aligned}
$$

where the loop-induced flavor violating $Z$ vertex is

$$
\begin{aligned}
F_{R}= & -\frac{N_{c}}{16 \pi^{2}} \sum_{q} y_{q e}^{*} y_{q \tau}\left(2 a_{R}^{q} C_{1 F}\left(x_{q}\right)-a_{L}^{q} C_{2 F}\left(x_{q}\right)\right. \\
& \left.-2 a^{L Q} C_{B}\left(x_{q}\right)+a_{R}^{l} B_{1}\left(x_{q}\right)\right),
\end{aligned}
$$

with $a_{L, R}^{q}, a_{R}^{l}$, and $a^{L Q}$ denoting the couplings of the $Z$-boson to quarks, leptons, and leptoquarks, $a^{i}=$ $-\frac{g}{\cos \theta_{W}}\left(T_{i}^{3}-e_{i} \sin ^{2} \theta_{W}\right)$, respectively.

The loop functions in (B9) read

$$
C_{1 F}(x)=\frac{1-x+(2-x) x \log x}{4(1-x)^{2}}-\frac{1}{4} \log \left(\frac{m_{R_{2}}^{2}}{Q^{2}}\right),
$$

$$
\begin{gathered}
C_{2 F}(x)=\frac{x-x^{2}+x \log x}{(1-x)^{2}}, \\
C_{B}(x)=\frac{x^{2}-x-x^{2} \log x}{4(1-x)^{2}}-\frac{1}{4} \log \left(\frac{m_{R_{2}}^{2}}{Q^{2}}\right), \\
B_{1}(x)=\frac{1}{4}\left(\frac{1-x^{2}+2 x^{2} \log x}{(1-x)^{2}}+2 \log \left(\frac{m_{R_{2}}^{2}}{Q^{2}}\right)-2\right) .
\end{gathered}
$$

We have checked that we can also reproduce the corresponding formulas given in [81], apart from $B_{1}$ which contains a trivial typo which can easily be seen by noting that $B_{1}$ has to be dimensionless. At first glance it might be surprising to see an explicit dependence on the renormalization scale $Q$. However, within one quark species one finds immediately that the terms proportional to $\log \left(m_{R_{2}}^{2} / Q^{2}\right)$ yield

$$
\begin{gathered}
\frac{y_{q e}^{*} y_{q \tau}}{2}\left(-a_{R}^{q}+a^{L Q}+a_{R}^{l}\right) \log \left(\frac{m_{R_{2}}^{2}}{Q^{2}}\right) \\
=\frac{y_{q e}^{*} y_{q \tau}}{2} T_{L Q}^{3} \log \left(\frac{m_{R_{2}}^{2}}{Q^{2}}\right) .
\end{gathered}
$$

Performing the sum over the $u$-type quarks, the CKMmatrix drops implying this part of the $Z$ penguin is indeed proportional to

$$
\log \left(\frac{m_{R_{2}^{2 / 3}}^{2}}{m_{R_{2}^{5 / 3}}^{2}}\right) \sum_{q=d, s, b}^{3} y_{q e}^{*} y_{q \tau} .
$$

We note for completeness that the ratio of the two leptoquark masses becomes 1 in the $S U(2)_{L}$ conserving limit in which this contribution vanishes as expected. Though not quite obvious from its form we note that $F_{R}$ also vanishes in the limit of infinite leptoquark masses.

\section{APPENDIX C: IMPLEMENTATION IN SARAH}

\section{Changes in SARAH}

In the context of this project, we have extended the functionality of SARAH to work with unbroken subgroups in order to implement the Pati-Salam model. We summarize the main parts of the SARAH model file and explain the new commands. For all details of the standard commands we refer to Refs. [42,83]. The following changes in SARAH have happened:

(1) The $S U(4)_{C}$ algebra was implemented to express the generators and structure constant of $S U(4)_{C}$ in terms of generators and structure constants of $S U(3)$ and Kronecker deltas. 
(2) The possibility to define unbroken subgroups of a bigger gauge group was added

(3) All necessary routines to write the matter and gauge fields, which are defined for the bigger group, in terms of the unbroken subgroup were developed
We tried to keep the changes in SARAH as generic as possible. That is, the new functionality is not restricted to the considered model or to Pati-Salam groups. However, we have tested the function thoroughly only for the model discussed in this paper. Therefore, one should be careful when using it with other models.

\section{The SARAH model files}

(1) The fundamental gauge groups $\left[S U(4)_{C} \times S U(2)_{L} \times U(1)_{R}\right]$ are defined as usual via the array Gauge:

$$
\begin{aligned}
& \text { Gauge }[[1]]=\{W R, U[1], \text { right, gR, True }\} \\
& \text { Gauge }[[2]]=\{W L, S U[2], \text { left , gL, True }\} ; \\
& \text { Gauge }[[3]]=\{P S, S U[4], \text { pati, g } 3, \operatorname{True}\} ;
\end{aligned}
$$

(2) In order to define that $S U(4)_{C}$ get broken to an unbroken group $S U(3)$, the following three steps are necessary: (a) The name of the group which shall be broken as well as the name of the unbroken subgroups are defined via UnbrokenSubgroups

$$
\text { UnbrokenSubgroups }=\{\text { pati }->\operatorname{col} \text { or }\}
$$

Here, the first part of the rule must correspond to an entry in Gauge.

(b) The features of the unbroken gauge groups in the new array AuxGauge are defined. This is completely analogue to the definition of a group in Gauge.

$$
\text { AuxGauge }=\{\{\mathrm{G}, \mathrm{SU}[3], \mathrm{color}, \mathrm{g} 3, \mathrm{False}\}\}
$$

The third entry must be identical to the chosen name in UnbrokenSubgroups.

(c) Names for the new gauge bosons must be introduced. The mapping between the fundamental gauge bosons $\left(V_{1} \cdots V_{N}\right)$ to a set of new gauge bosons $\left\{V^{a}, V^{b}, \ldots, V^{x}\right\}$ with dimensions $\left\{N_{a}, N_{b}, \ldots, N_{b}\right\}$ under the unbroken subgroup is done as

$$
\left(\begin{array}{c}
V_{1} \\
V_{2} \\
V_{3} \\
\cdot \\
\cdot \\
\cdot \\
V_{N-1} \\
V_{N}
\end{array}\right)=\left(\begin{array}{c}
V_{1}^{a} \\
\cdots \\
V_{N_{a}}^{a} \\
V_{1}^{b} \\
\cdots \\
V_{N_{a}}^{b} \\
\cdot \\
\cdot \\
\cdot \\
V_{1}^{x} \\
\cdots \\
V_{N_{a}}^{x}
\end{array}\right) .
$$

This relation is defined in the model file using the new array RepGaugeBosons. For each unbroken subgroup a list must be given which consists of pairs of the name of a gauge boson and its dimension. 


$$
\text { RepGaugeBosons }=\{\{\{V G, 8\},\{V X, 3\},\{V Y, 3\},\{V S, 1\}\}\}
$$

Note, the names for the gauge bosons must always start with V. From this definition, the mapping of the ghost is also derived. The names of the ghost fields are those of the vector boson with $\mathrm{V}$ replaced by $\mathrm{g}$.

(3) After the definition of the gauge groups, the matter fields are defined. This is done for nonsupersymmetric fields using the arrays Fermionfield and ScalarField. For fields which transform nontrivially under the broken gauge groups, the tensor notation is used. Thus, the fundamental representation is a vector of dimension $N$. If the unbroken subgroup has dimension $n$, the relation between the components of the fields are

$$
\left(\begin{array}{c}
\Phi_{1} \\
\Phi_{2} \\
\cdots \\
\Phi_{N-1} \\
\Phi_{N}
\end{array}\right)=\left(\begin{array}{c}
\Psi_{1} \\
\cdots \\
\Psi_{n} \\
\Psi^{\prime} \\
\cdots \\
\Psi^{\prime \cdots^{\prime}}
\end{array}\right)
$$

The number of fields with a prime is $N-n$.

For the adjoint representation, an $N \times N$ matrix is used. This matrix is then decomposed as

$$
\left(\begin{array}{ccc}
\Phi_{11} & \cdots & \Phi_{1 N} \\
\vdots & & \vdots \\
\Phi_{N 1} & \cdots & \Phi_{N N}
\end{array}\right)=\left(\begin{array}{cccccc}
\Psi_{11} & \cdots & \Psi_{1 n} & \Psi_{1}^{\prime} & \ldots & \Psi_{1}^{\prime \cdots \prime} \\
\vdots & & \vdots & \vdots & & \vdots \\
\Psi_{n 1} & \ldots & \Psi_{n n} & \Psi_{n}^{\prime} & \ldots & \Psi_{n}^{\prime \cdots \prime} \\
\tilde{\Psi}_{1}^{\prime} & \ldots & \tilde{\Psi}_{n}^{\prime} & \alpha^{\prime} & \cdots & \phi^{\prime \cdots \prime} \\
\vdots & & \vdots & \vdots & & \vdots \\
\tilde{\Psi}_{1}^{\prime \cdots \prime} & \ldots & \tilde{\Psi}_{n}^{\prime} & \omega^{\prime} & \cdots & \omega^{\prime \cdots \prime}
\end{array}\right) .
$$

Here, $\Psi$ is in the adjoint representation of the unbroken subgroup and all primed fields $\Psi^{\prime}$ and $\tilde{\Psi}$ are vectors under the unbroken subgroup. The fields $\alpha$ to $\omega$ are singlets under the unbroken group.

(a) In the given model, the fermion fields are either singlets or transform in the (anti)fundamental representation.

This is defined via

$$
\begin{aligned}
& \text { Fermionfields }[[1]]= \\
& \{F Q L, 3,\{\{u L[\operatorname{col} o r, 3], v L\},\{d L[\operatorname{col} \circ r, 3], e L\}\}, 0,2,4\} \text {; } \\
& \text { Fermionfields [[2] ] = } \\
& \{\mathrm{FU}, 3,\{\mathrm{uR}[\mathrm{color},-3], \mathrm{vR}\},-1 / 2,1,-4\} \text {; } \\
& \text { Fermionfields [3] ] = } \\
& \{F D, 3,\{d R[\operatorname{col} \circ r,-3], e R\}, 1 / 2,1,-4\} \text {; } \\
& \text { Fermionfields }[4]=\{\mathrm{Si}, 3, \mathrm{Sing}, 0,1,1\} \text {; }
\end{aligned}
$$

Note that here the last three entries define the representation with respect to the gauge groups defined in Gauge. The representation with respect to the unbroken subgroup are defined for each component field in squared brackets, i.e., uL [color, 3] means that the field uL is a color triplet.

(b) In the scalar sector, the adjoint representation is needed in addition. All scalars are defined via 


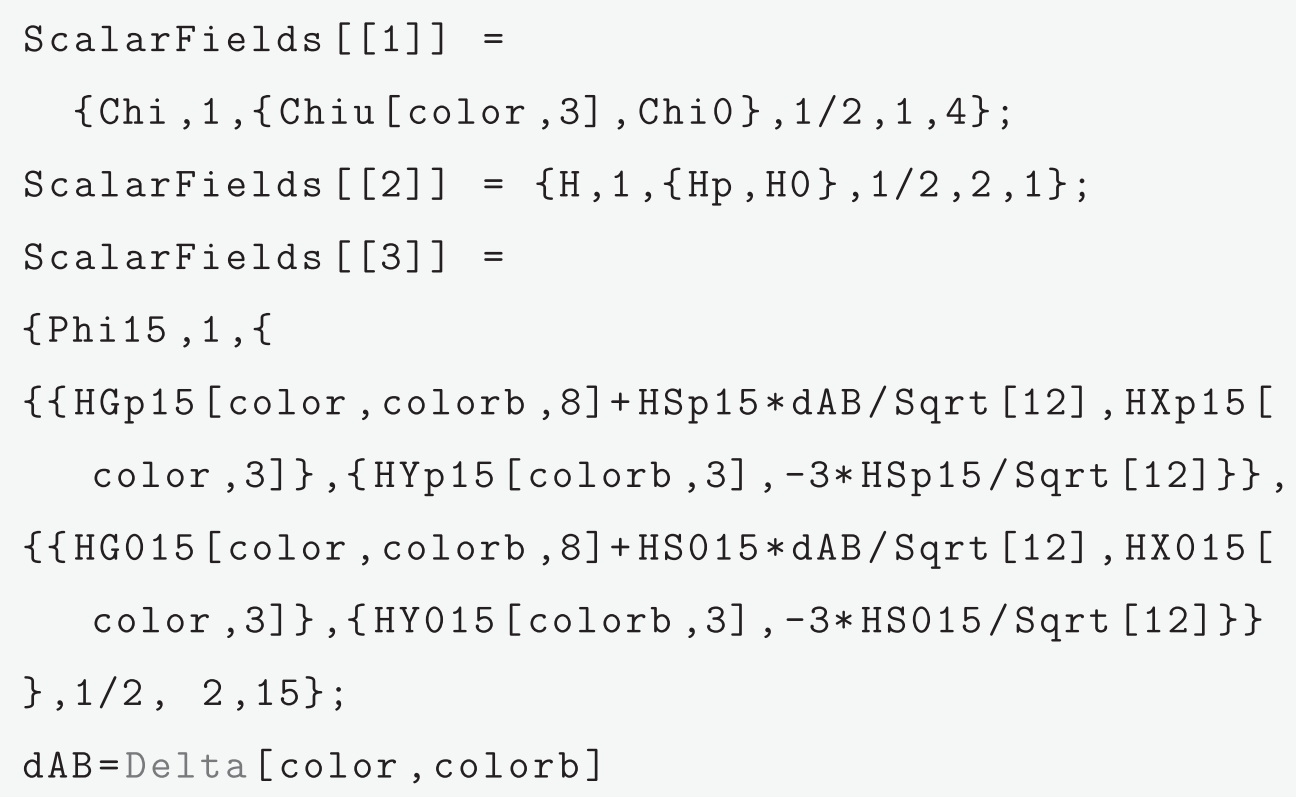

Here, we have introduced the abbreviation $\mathrm{AAB}$ only for better readability. Note, that for the tensor representation the name of the second color index is extended by b (i.e., colorb to prevent any ambiguity).

There is one additional subtlety: in SARAH and other codes such as MadGraph, CalcHep, or WHIZARD the higher dimensional representations of unbroken gauge groups, i.e., the color group, are not written as tensors but vectors. Therefore, it is necessary to rewrite the neutral and charged octets. The necessary definitions are given in the list TensorRepToVector which reads in our case

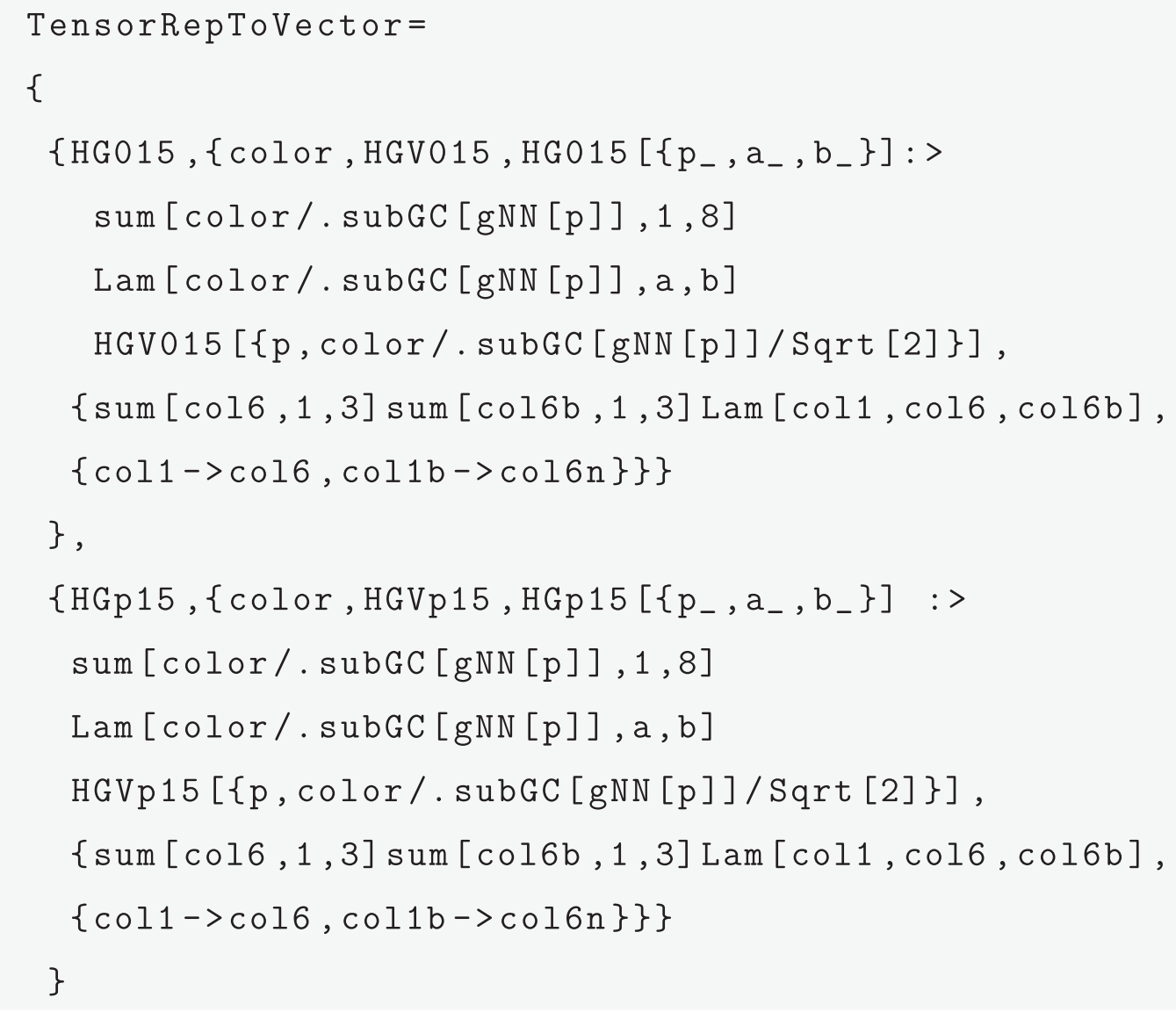




$$
\begin{aligned}
& \} ; \\
& \text { gNN }\left[g_{-}\right]:= \\
& \qquad 5+\text { ToExpression [StringTake [ToString }[g],\{-1\}]] ;
\end{aligned}
$$

Each entry consists of the following pieces:

(i) The name of tensor field (HG015, HGp15).

(ii) The name of the gauge group for which the rewriting shall take place (color).

(iii) The name which should be used for the vector representation (HGV015, HGVp15).

(iv) The substitution rule:

$$
\begin{aligned}
& \text { HG015 [ } \left.\left\{p_{-}, a_{-}, b_{-}\right\}\right] \text {: }> \\
& \quad \text { sum [color/.subGC [gNN [p] ], 1, 8] Lam [color/. subGC [gNN [p] ] , a, b] } \\
& \text { HGV015[\{p, color/.subGC[gNN [p] ]/Sqrt [2] }\}]
\end{aligned}
$$

Here, $\mathrm{p}$ is a unique index (gen1, gen2, gen3, gen4) counting the fields in each interaction term, and gNN is a function to shift this index by 5 . Moreover, $a, b$ are the color indices. Therefore, the above line is interpreted as

$$
\Phi_{\alpha \beta}^{p} \rightarrow \sum_{f(p)} \lambda_{\alpha \beta}^{f(p)} \frac{1}{\sqrt{2}} \tilde{\Phi}_{f(p)}^{p}
$$

with a function $f$ to rename the indices.

(v) Finally, one needs to define also the reverse operation, i.e., the relation to rewrite the vector into the tensor representation. This is needed to derive the ghost interactions.

(4) Once the gauge sector and relation for the fields before electroweak symmetry breaking (EWSB) are fixed, the rest of the model file is straightforward and follows the standard SARAH conventions:

(a) Lagrangian: The Lagrangian consists of two parts:

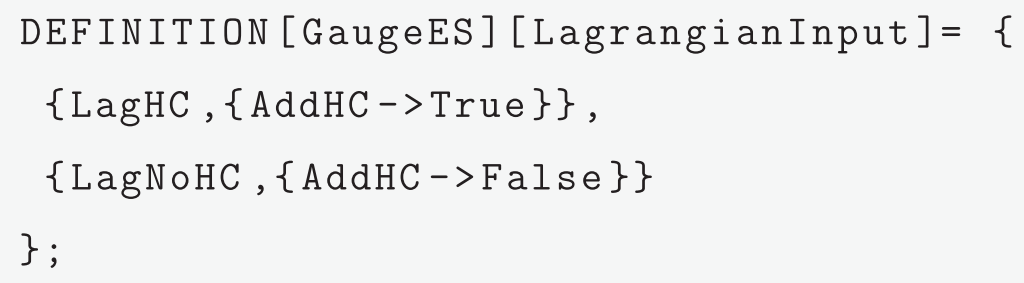

For the first part, the Hermitian conjugate needs to be added (AddHC->True). This part involves the fermion interactions as well as $\lambda_{4}$ :

$$
\begin{aligned}
& \text { LagHC }=-(Y 1 \text { FQL.FU.H }+ \text { Y2 FQL.FU.Phi15 } \\
&+ \text { Y3 conj[H].FQL.FD + Y4 conj[Phi15].FQL.FD } \\
&+ \text { Y5 FU.Chi.Si }+\backslash[M u / 2 \text { Si.Si } \\
&+ \text { lambda4 conj[H].conj[Chi].Phi15.Chi }) ;
\end{aligned}
$$

All other parts of the Lagrangian are already Hermitian and are defined via 


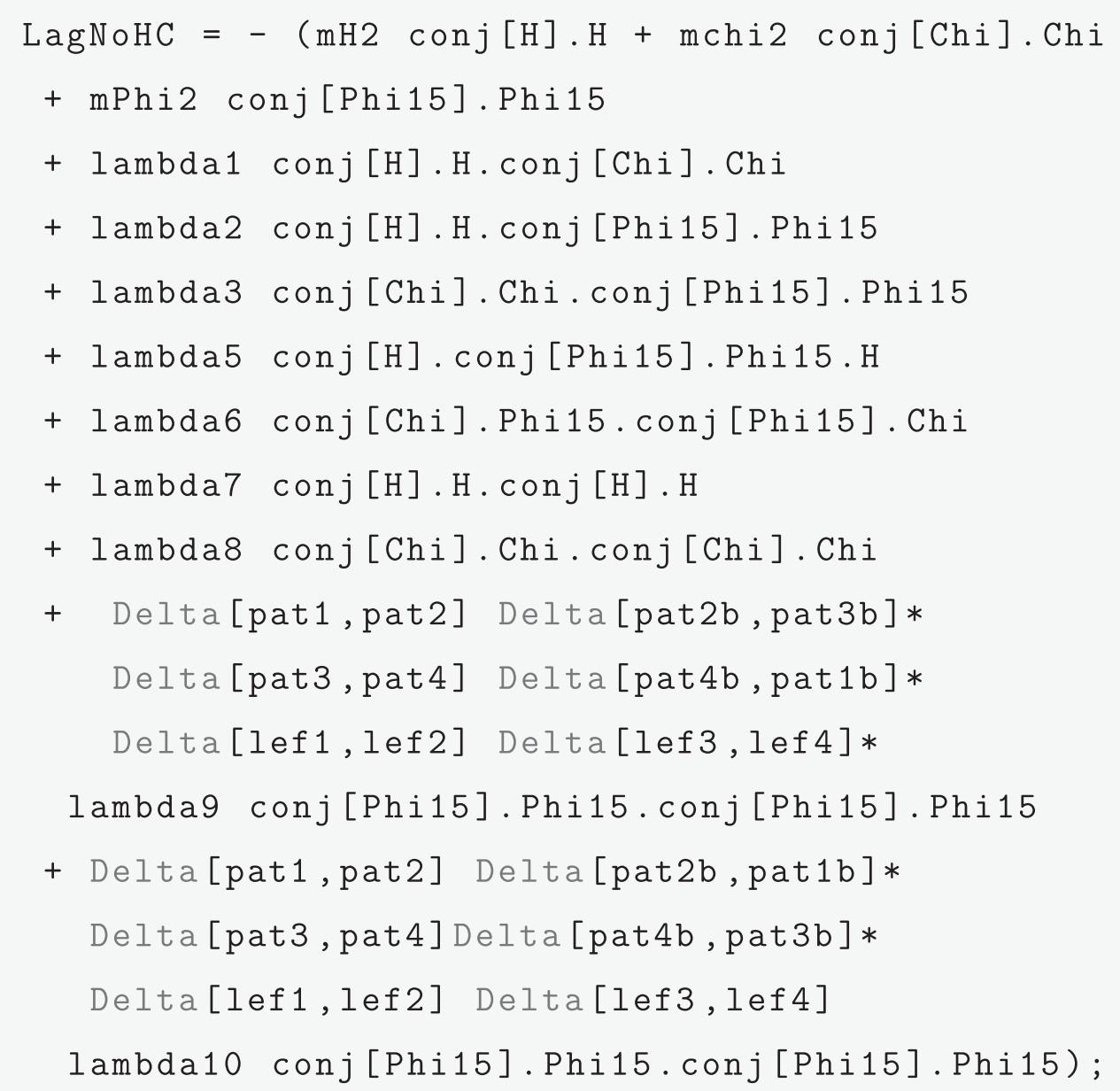

For all terms but $\lambda_{9}$ and $\lambda_{10}$ the index contraction is unique. For those terms one needs to define the contraction explicitly using Kronecker deltas. The remaining terms coming with $\lambda_{11}-\lambda_{19}$ can be implemented in a similar fashion.

(b) VEVs: The VEVs are set via

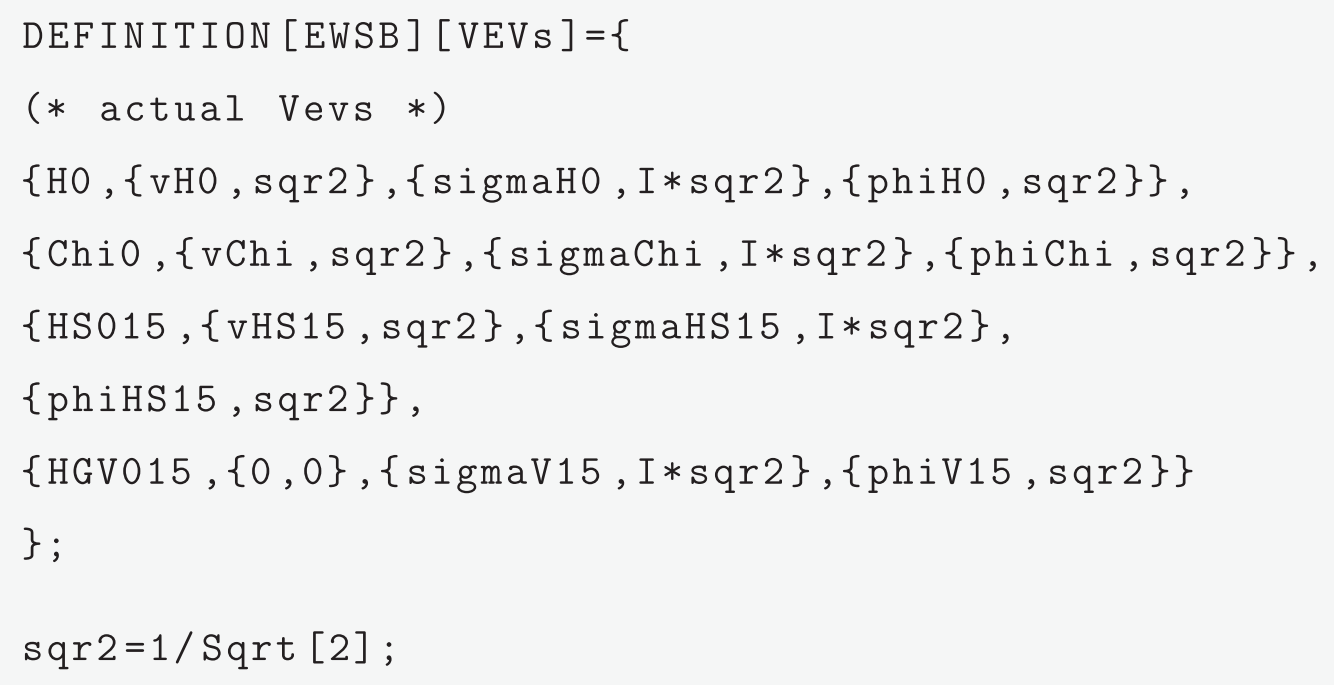


Although the color octet does not receive a VEV, its $C P$ even and odd component has a different mass. Therefore, it is also decomposed in real fields.

(c) Gauge bosons: The rotations of the gauge bosons are defined via

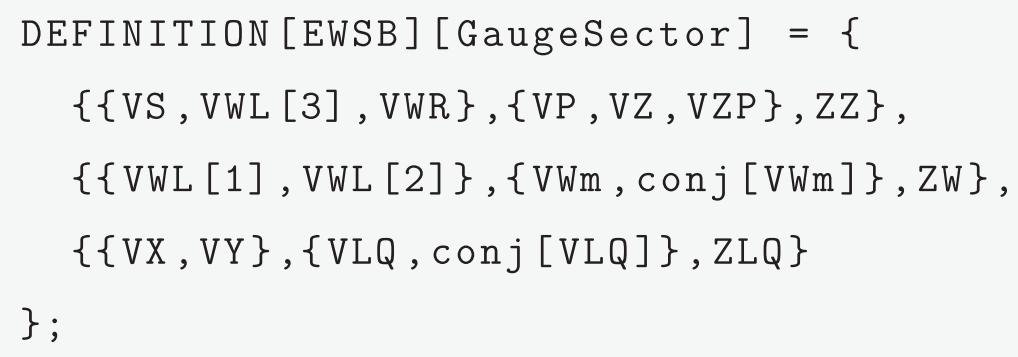

with the rotation matrices defined in the parameters.m file as

$$
Z^{W}=Z^{L Q}=\frac{1}{\sqrt{2}}\left(\begin{array}{cc}
1 & 1 \\
i & -i
\end{array}\right)
$$

For the rotation in the neutral sector no explicit parametrization for $\mathrm{ZZ}$ is used.

(d) Matter fields: The rotations in the matter sector are defined via

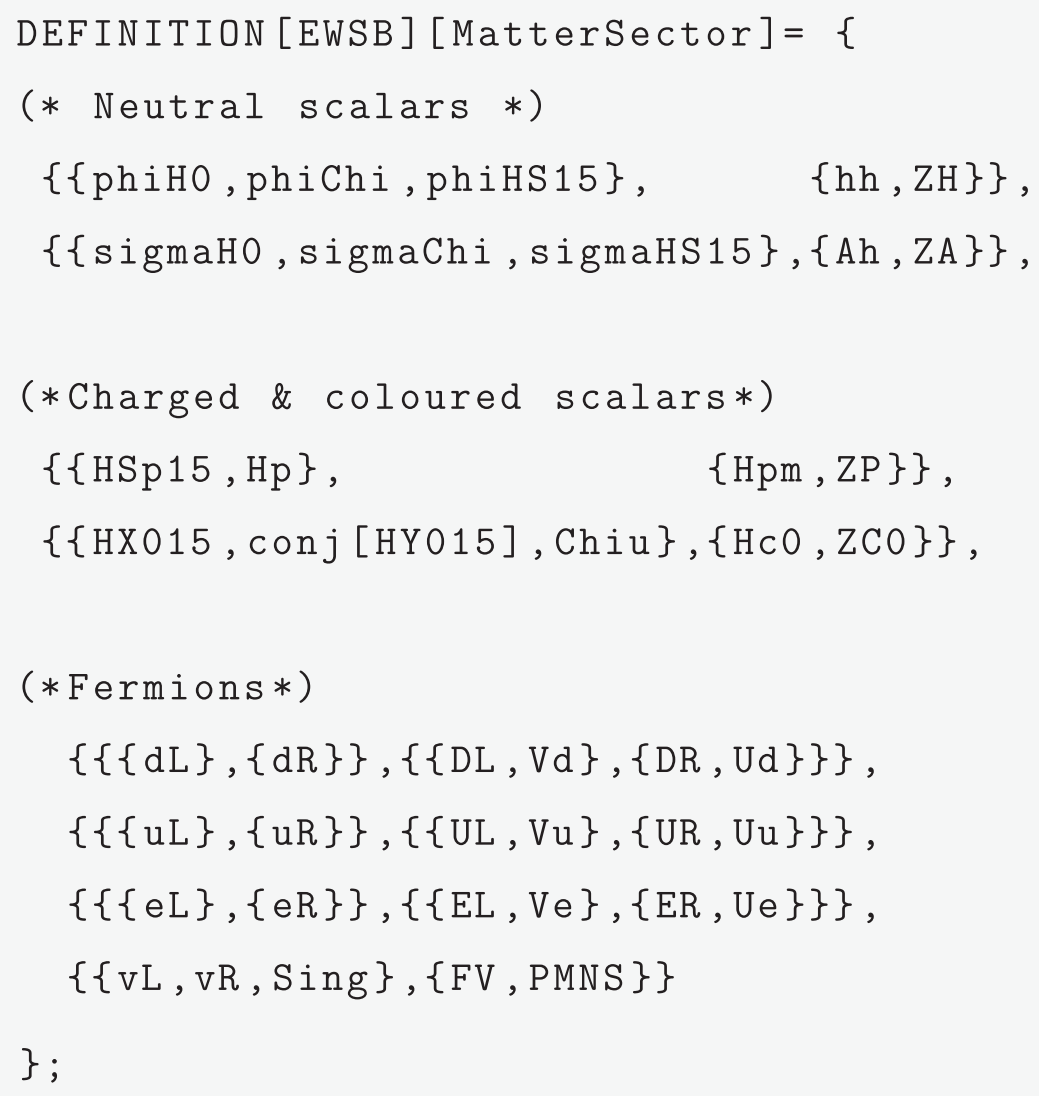


(e) Dirac spinors: The Weyl spinors are combined to Dirac spinors via

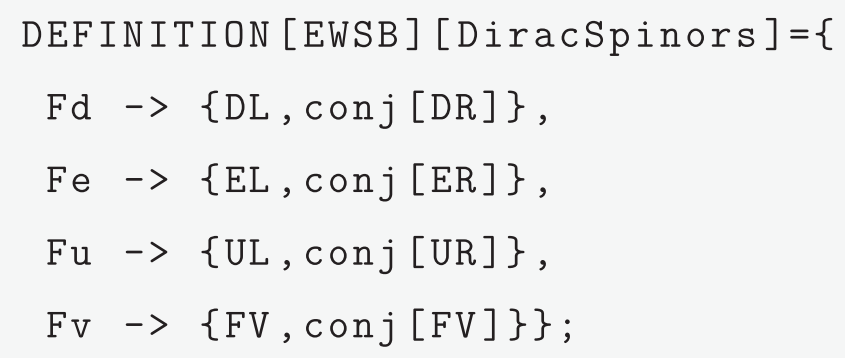

Useful relations for the generators used by SARAH are

$$
\begin{gathered}
\Lambda_{y 4}^{8+x}=\Lambda_{4 y}^{8+x}=\frac{1}{2} \delta_{x y}, \\
\Lambda_{y 4}^{11+x}=-\Lambda_{4 y}^{11+x}=-\frac{1}{2} i \delta_{x y},
\end{gathered}
$$

for $1 \leq x, y \leq 3$.

For the structure constants, one can make use of

$$
\begin{gathered}
F_{a b(c+8)}=F_{a b(c+11)}=0, \\
F_{i a b} F_{i c d}=f_{i a b} f_{i c d}, \\
F_{c(8+x)(8+y)}=F_{c(11+x)(11+y)}=i \frac{1}{4}\left(\lambda_{x y}^{c}-\left(\lambda_{x y}^{c}\right)^{*}\right),
\end{gathered}
$$

$$
\begin{gathered}
F_{c(8+x)(11+y)}=-\frac{1}{4}\left(\lambda_{x y}^{c}+\left(\lambda_{x y}^{c}\right)^{*}\right), \\
F_{(8+x)(8+y) 15}=F_{(8+x)(8+y)(8+z)}=F_{(8+x)(8+y)(11+z)} \\
=F_{(8+x)(11+y)(11+z)}=F_{(11+x)(11+y)(11+x)} \\
=F_{(11+x)(11+y) 15}=0 \\
F_{(8+x)(11+y) 15}=\sqrt{\frac{2}{3}} \delta_{x y},
\end{gathered}
$$

for $1 \leq a, b, c \leq 8$ and $1 \leq x, y, z \leq 3$

[1] A. Matyja et al. (Belle Collaboration), Phys. Rev. Lett. 99, 191807 (2007).

[2] A. Bozek et al. (Belle Collaboration), Phys. Rev. D 82, 072005 (2010).

[3] R. Aaij et al. (LHCb Collaboration), Phys. Rev. Lett. 113, 151601 (2014).

[4] M. Huschle et al. (Belle Collaboration), Phys. Rev. D 92, 072014 (2015).

[5] R. Aaij et al. (LHCb Collaboration), Phys. Rev. Lett. 115, 111803 (2015); 115, 159901(E) (2015).

[6] R. Aaij et al. (LHCb Collaboration), J. High Energy Phys. 08 (2017) 055.

[7] R. Aaij et al. (LHCb Collaboration), Phys. Rev. Lett. 122, 191801 (2019).

[8] M. Bauer and M. Neubert, Phys. Rev. Lett. 116, 141802 (2016).

[9] M. Bauer and M. Neubert, Phys. Rev. D 93, 115030 (2016).

[10] W. Chao, Nucl. Phys. B911, 231 (2016).

[11] C. W. Murphy, Phys. Lett. B 757, 192 (2016).
[12] R. Alonso, B. Grinstein, and J. Martin Camalich, J. High Energy Phys. 10 (2015) 184.

[13] L. Calibbi, A. Crivellin, and T. Ota, Phys. Rev. Lett. 115, 181801 (2015).

[14] S. Fajfer and N. Košnik, Phys. Lett. B 755, 270 (2016).

[15] G. Hiller, D. Loose, and K. Schönwald, J. High Energy Phys. 12 (2016) 027.

[16] F. F. Deppisch, S. Kulkarni, H. Päs, and E. Schumacher, Phys. Rev. D 94, 013003 (2016).

[17] B. Bhattacharya, A. Datta, J.-P. Guévin, D. London, and R. Watanabe, J. High Energy Phys. 01 (2017) 015.

[18] R. Barbieri and A. Tesi, Eur. Phys. J. C 78, 193 (2018).

[19] D. Buttazzo, A. Greljo, G. Isidori, and D. Marzocca, J. High Energy Phys. 11 (2017) 044.

[20] A. K. Alok, B. Bhattacharya, A. Datta, D. Kumar, J. Kumar, and D. London, Phys. Rev. D 96, 095009 (2017).

[21] J. Kumar, D. London, and R. Watanabe, Phys. Rev. D 99, 015007 (2019).

[22] A. Biswas, A. K. Swain, and A. Shaw, Lett. High Energy Phys. 2, 126 (2019). 
[23] A. Crivellin, D. Müller, and T. Ota, J. High Energy Phys. 09 (2017) 040.

[24] A. Crivellin, D. Mueller, A. Signer, and Y. Ulrich, Phys. Rev. D 97, 015019 (2018).

[25] L. Di Luzio, A. Greljo, and M. Nardecchia, Phys. Rev. D 96, 115011 (2017).

[26] L. Calibbi, A. Crivellin, and T. Li, Phys. Rev. D 98, 115002 (2018).

[27] M. Bordone, C. Cornella, J. Fuentes-Martin, and G. Isidori, Phys. Lett. B 779, 317 (2018).

[28] I. Doršner, S. Fajfer, D. A. Faroughy, and N. Košnik, J. High Energy Phys. 10 (2017) 188.

[29] N. Assad, B. Fornal, and B. Grinstein, Phys. Lett. B 777, 324 (2018).

[30] M. Blanke and A. Crivellin, Phys. Rev. Lett. 121, 011801 (2018).

[31] A. Greljo and B. A. Stefanek, Phys. Lett. B 782, 131 (2018).

[32] M. Bordone, C. Cornella, J. Fuentes-Martín, and G. Isidori, J. High Energy Phys. 10 (2018) 148.

[33] S. Matsuzaki, K. Nishiwaki, and K. Yamamoto, J. High Energy Phys. 11 (2018) 164.

[34] J. Heeck and D. Teresi, J. High Energy Phys. 12 (2018) 103.

[35] S. Balaji, R. Foot, and M. A. Schmidt, Phys. Rev. D 99, 015029 (2019).

[36] B. Fornal, S. A. Gadam, and B. Grinstein, Phys. Rev. D 99, 055025 (2019).

[37] I. Doršner, S. Fajfer, A. Greljo, J. F. Kamenik, and N. Košnik, Phys. Rep. 641, 1 (2016).

[38] T. Faber, M. Hudec, M. Malinský, P. Meinzinger, W. Porod, and F. Staub, Phys. Lett. B 787, 159 (2018).

[39] A. D. Smirnov, Phys. Lett. B 346, 297 (1995).

[40] P. Fileviez Perez and M. B. Wise, Phys. Rev. D 88, 057703 (2013).

[41] R. Aaij et al. (LHCb Collaboration), J. High Energy Phys. 02 (2016) 104.

[42] F. Staub, arXiv:0806.0538.

[43] F. Staub, Comput. Phys. Commun. 181, 1077 (2010).

[44] F. Staub, Comput. Phys. Commun. 182, 808 (2011).

[45] F. Staub, Comput. Phys. Commun. 184, 1792 (2013).

[46] F. Staub, Comput. Phys. Commun. 185, 1773 (2014).

[47] W. Porod, Comput. Phys. Commun. 153, 275 (2003).

[48] W. Porod and F. Staub, Comput. Phys. Commun. 183, 2458 (2012).

[49] J. Alwall, M. Herquet, F. Maltoni, O. Mattelaer, and T. Stelzer, J. High Energy Phys. 06 (2011) 128.

[50] J. Alwall, C. Duhr, B. Fuks, O. Mattelaer, D. G. Öztürk, and C.-H. Shen, Comput. Phys. Commun. 197, 312 (2015).

[51] R. N. Mohapatra and J. W. F. Valle, Phys. Rev. D 34, 1642 (1986).

[52] A. D. Smirnov, Mod. Phys. Lett. A 33, 1850019 (2018).

[53] R. M. Fonseca, J. Phys. Conf. Ser. 873, 012045 (2017).

[54] G. D’Amico, M. Nardecchia, P. Panci, F. Sannino, A. Strumia, R. Torre, and A. Urbano, J. High Energy Phys. 09 (2017) 010.
[55] D. Bečirević and O. Sumensari, J. High Energy Phys. 08 (2017) 104.

[56] S. Fajfer, N. Košnik, and L. Vale Silva, Eur. Phys. J. C 78, 275 (2018).

[57] P. Yu. Popov, A. V. Povarov, and A. D. Smirnov, Mod. Phys. Lett. A 20, 3003 (2005).

[58] M. Tanabashi et al. (Particle Data Group), Phys. Rev. D 98, 030001 (2018).

[59] S. Bertolini, L. Di Luzio, and M. Malinsky, Phys. Rev. D 87, 085020 (2013).

[60] W. Porod, F. Staub, and A. Vicente, Eur. Phys. J. C 74, 2992 (2014).

[61] E. Arganda, M. J. Herrero, and J. Portoles, J. High Energy Phys. 06 (2008) 079.

[62] A. Lami, J. Portoles, and P. Roig, Phys. Rev. D 93, 076008 (2016).

[63] M. Jamin, A. Pich, and J. Portoles, Phys. Lett. B 664, 78 (2008).

[64] D. M. Straub, arXiv:1810.08132.

[65] J. Aebischer et al., Comput. Phys. Commun. 232, 71 (2018).

[66] W. Altmannshofer et al. (Belle-II Collaboration), arXiv: 1808.10567.

[67] M. Aaboud et al. (ATLAS Collaboration), New J. Phys. 18, 093016 (2016).

[68] A. M. Sirunyan A. Tumasyan, W. Adam et al., J. High Energy Phys. 03 (2019) 170.

[69] M. Drees, H. Dreiner, D. Schmeier, J. Tattersall, and J. S. Kim, Comput. Phys. Commun. 187, 227 (2015).

[70] D. Dercks, N. Desai, J. S. Kim, K. Rolbiecki, J. Tattersall, and T. Weber, Comput. Phys. Commun. 221, 383 (2017).

[71] I. V. Frolov, M. V. Martynov, and A. D. Smirnov, Mod. Phys. Lett. A 31, 1650224 (2016).

[72] M. V. Martynov and A. D. Smirnov, EPJ Web Conf. 158, 02009 (2017).

[73] M. I. Gresham and M. B. Wise, Phys. Rev. D 76, 075003 (2007).

[74] N. Arkani-Hamed, T. Han, M. Mangano, and L.-T. Wang, Phys. Rep. 652, 1 (2016).

[75] R. Contino et al., CERN Yellow Report No. 255 (2017), https://doi.org/10.23731/CYRM-2017-003.255.

[76] A. Hayreter and G. Valencia, arXiv:1810.04048.

[77] A. M. Sirunyan et al. (CMS Collaboration), Phys. Rev. Lett. 120, 201801 (2018).

[78] A. M. Sirunyan et al. (CMS Collaboration), J. High Energy Phys. 08 (2018) 130.

[79] D. Goncalves-Netto, D. Lopez-Val, K. Mawatari, T. Plehn, and I. Wigmore, Phys. Rev. D 85, 114024 (2012).

[80] M. Aaboud et al. (ATLAS Collaboration), J. High Energy Phys. 11 (2018) 085.

[81] E. Arganda and M. J. Herrero, Phys. Rev. D 73, 055003 (2006).

[82] A. Crivellin, M. Hoferichter, and P. Schmidt-Wellenburg, Phys. Rev. D 98, 113002 (2018).

[83] F. Staub, Adv. High Energy Phys. 2015, 840780 (2015). 